\title{
Synthesis, reactivity, and electronic structure of multifarious, five-membered heteroaryl and heteroaroyl azides
}

\author{
Paolo Zanirato \\ Dip.to di Chimica Organica 'A. Mangini', Università di Bologna, Viale Risorgimento 4, \\ Bologna, 40136, Italy \\ E-mail: paolo.zanirato@unibo.it
}

\begin{abstract}
The aim of this account is to review our most important outcomes - published during the last 30 years - concerning the azido group that is linked to five-membered heteroaryl and heteroaroyl systems. The main focus of this manuscript is on the 'azido transfer' reaction to heteroaryl azides, and the peculiar thermal and chemical reactivities of these precursor species. In particular, the following topics are considered: (a) the ring cleavage of $\alpha$-heteroaryl azides to form 4-cyano-1,3-heterodienes, (b) the reactivity of thermally generated $\beta$-nitrene, (c) the 1,3dipolar cycloaddition of the azido group to various terminal or 1,2-disubstituted alkenes or alkynes to form nitrogen-containing biheterocycles, (d) the generation of electrophile nitrenium ions with Lewis acids, (e) the conversion of heteroaroyl azides into isocyanates, and (f) the 1,3dipolar cycloadditions of heteroaroyl azides with 'activated' olefins. The concurrence and discordance of the thermal and chemical behaviour between heteroaryl azides, and the related substituted phenyl azides will be critically considered here.
\end{abstract}

Keywords: Heteroaryl azides, heteroaroyl azides, heteroaryl nitrenium ions, heteroaryl nitrenes, synthesis and reaction mechanisms, heteroaryl ring cleavage, 1,3-dipolar cycloaddition, MS spectroscopy

\section{Contents}

1. Introduction

2. Five Membered Heteroaryl Azides

2.1. Synthesis via 'azido transfer'

2.2. General reactivity of heteroaryl azides

2.3. Heteroaryl amines by the reduction of the azido group

2.4. Thermal dissociation of heteroaryl azides

2.5. Nitrenium ions via azido fragmentation with boron derivatives 
2.6. 1,3-Dipolar cycloadditions of heteroaryl azides

3. Five-membered Azido(heteroaryl)methanones

3.1. General synthesis and Curtius Rearrangement

3.2. 1,3-Dipolar cycloadditions involving carbonyl azides

4. Conclusions and Outlook

Acknowledgements

References

\section{Introduction}

The chemical behaviour of the covalent azido group $\left(-\mathrm{N}_{3}\right)$ strongly depends on its electronic structure, which in a ground state, is characterised by three nitrogen atoms linearly linked by bonding and nonbonding electron pairs. The great synthetic utility of this group is generally attributed to the energetic weight of its structure, that is, a combination of dipolar contributing resonance forms, as well as by the nature of the carrying substrate. The broad thermo-chemical behaviours of the organic azides are illustrated in Fig. 1.

i) 'Fragility'; thermal or photochemical dissociation to nitrene (S or T) and molecular nitrogen:

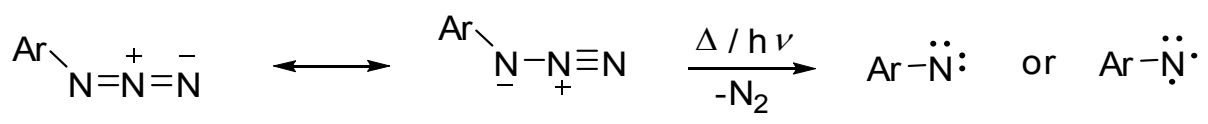

ii) 'Flexibility'; generation of 1,3-dipolar allyl type bent from linear propargyl allenyl structures:

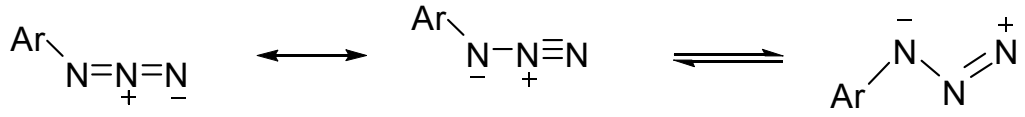

iii) 'Disposability'; either with electrophiles $\left(\mathrm{E}^{+}\right)$or nucleophiles $\left(\mathrm{Nu}^{-}\right)$to form nitrenium ions $(\mathrm{I})$ or triazenes (II), respectively:

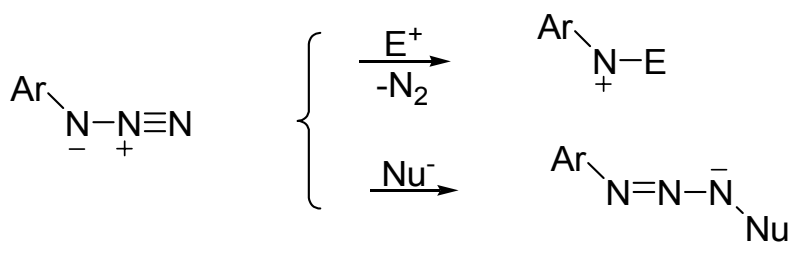

\section{Figure 1}

Many organic and inorganic compounds containing one (or more) of the azido group are known for their thermal- or shock-sensitivity. ${ }^{1}$ For this reason, preventive thermal analysis, performed by differential scanning calorimetry (DSC) and/or by thermal gravimetry (TGA), and C-80 calorimetric analysis, may be advisable. Some papers discussing the most likely hazards 
related to the thermal decomposition of some aryl ${ }^{2 \mathrm{a}}$ and heteroaroyl ${ }^{2 \mathrm{~b}, \mathrm{c}}$ azides have been recently published.

Aryl azides are long-lasting and known for their ease of preparation and transformation into various substituents (amine, triazene, aza-ylide, and isocyanate), reactive species (nitrene, nitrenium ion), and nitrogen-containing heterocycles (azirine, aziridine, triazole, triazoline, and azole), and for civil and industrial applications (propellants, explosives, polymer cross-linkers, rubber vulcanisations, reactive dyes, blowing agents, and biological and pharmaceutical active compounds). , $3 \mathrm{a}-\mathrm{h}$

The thermal decomposition (TD) of simple phenyl or aryl azides, under 'controlled' conditions occurs with loss of molecular nitrogen and give rise to a great variety of products; which are generally dependent on the medium, substrate, substituent(s), and/or initial source of energy (thermal, chemical, or photochemical). ${ }^{3 \mathrm{a}-\mathrm{c}}$ The nitrene intermediate, generated by TD, can exist in different electronic ground states, that is, singlet and/or triplet states $\left(\mathrm{S}_{0}\right.$ and/or $\left.\mathrm{T}_{1}\right)$. This significantly differentiates between both the chemical outcomes and reactivities (intramolecular rearrangement, intermolecular $\mathrm{C}-\mathrm{H}$ and $\mathrm{N}-\mathrm{H}$ insertion, radical coupling, and chelation) of the two electronic species. ${ }^{3}$

The last three decades have seen an overwhelming interest in the synthesis of novel heteroaryl azides, and their use as starting materials for transformation into various and different substituents, reactive species, and nitrogen-containing heterocycles. ${ }^{4}$ At this stage, the primary aim of this accounts, which chiefly covers the last 30 years of our work on the five-member heteroaryl and heteroaroyl azides, is to familiarise newcomers with the more important results in these fields.

\section{Five membered heteroaryl azides}

\subsection{Synthesis via 'azido transfer'}

Azides derived from five-membered heteroaryls play an ever-increasing role in chemistry, and a number of the reactions applied to these compounds will be discussed here. Our long-term interest in this field started with the application of the synthetic method proposed by Smith to heteroaryl systems to produce aryl azides, ${ }^{5}$ that is, the 'azido transfer' protocol. The protocol is based on the reaction of an aryl Grignard reagent with $p$-toluenesulphonyl azide (tosyl azide) to form a triazene-salt intermediate that can be fragmented into aryl azide and $\mathrm{MgBr}$ tosylate in a buffered solution of tetrasodium pyrophosphate $\left(\mathrm{Na}_{4} \mathrm{P}_{2} \mathrm{O}_{7}\right)$. (see Scheme 1)

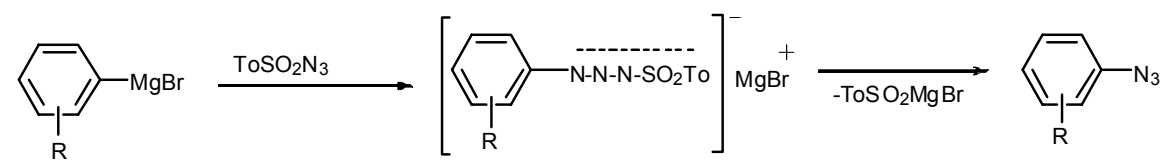

\section{Scheme 1}


We initially extended the modified Smith protocol to the syntheses of 2-azido and 3-azidoheteroaryls using 2-lithium- and 3-lithium-thiophene, ${ }^{6 \mathrm{a}}$ and 2-lithium- ${ }^{6 \mathrm{~b}}$ and 3-lithiumbenzothiophene, instead of the corresponding Grignard salts. These organolithium derivatives, obtained in a diethyl ether dry medium with $1.6 \mathrm{M} n$-butyllithium (unless otherwise stated), react with the electrophilic terminal nitrogen atom of the tosyl azide to give the appropriate lithium triazene solid salts generally in almost perfect quantitative yields (see Scheme 3). Regioselectivity between the 3- and 2-positions on the heterocyclic ring is effectively ensured by means of either halogen-lithium or proton-lithium exchange, (Scheme 2, and Eq. 1 and Eq. 2, respectively). ${ }^{7}$

Eq. 1
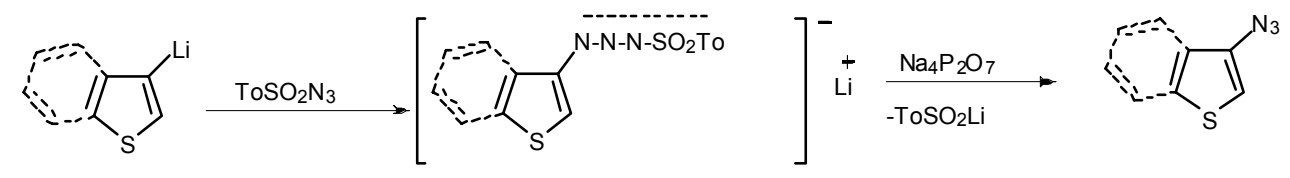

Eq. 2
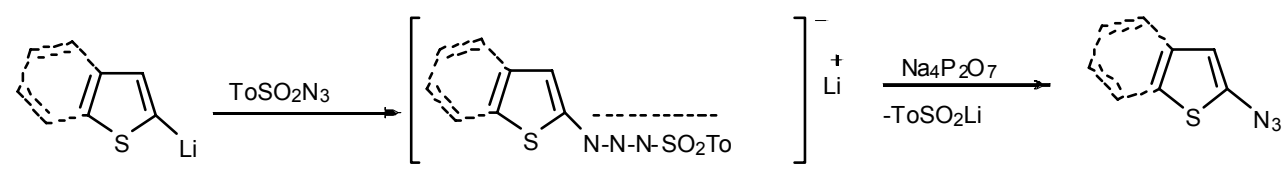

\section{Scheme 2}

Alternatively, these triazene lithium salts can also be used as starting materials to synthesise 1,3,3-trisubstituted triazenes, ${ }^{8}$ which have applications in cancer therapy ${ }^{9}$ and many other areas. $^{10}$

We used the lithium azido transfer protocol by which stable 3-azido-thiophene and benzo[b]thiophene (3-BTA) are obtained in good yields. Unfortunately, the 2-azides, namely 2azido-thiophene and -benzo $[b]$ thiophene (2-BTA), are obtained at lower yields, due to their instability even if stored at low temperatures. Accordingly, sodium pyrophosphate-induced fragmentation of the triazene lithium salts of the latter species to the corresponding azides is conducted at lower temperatures (i.e., between -5 and $0^{\circ} \mathrm{C}$ ) than those employed for the preparation of the 3 -azido analogues (namely, $25^{\circ} \mathrm{C}$ ).

Although organolithium chemistry represents a facile and convenient general route to the synthesis of hundreds of heteroarenes; the number of heteroaryl azides prepared to date via the 'azido transfer' protocol is comparatively low. This complements the most commonly employed methods for obtaining organic azides, ${ }^{11}$ some of which are unsuited for the preparation of the simple heteroaryl azides (i.e. $\mathrm{S}_{\mathrm{N}} \mathrm{Ar}$, instability of certain aminoheteroaryls). Later, the standardised lithium 'azido transfer' protocol was extended to the synthesis of simple azidoheteroaryls and their benzo-fuse derivatives, such as selenophene, ${ }^{12} \mathrm{~N}$-methylpyrrole, ${ }^{13}$ indole, ${ }^{14}$ benzofuran, ${ }^{15} \mathrm{~N}$-methylindole, ${ }^{16}$ and several substituted azido thiophenes containing methyl, trimethylsilyl, acetyl, or 2,2,2-trifluoroacetyl groups. ${ }^{17 a, b}$ 
Remarkably, isomeric acetyl or 2,2,2-trifluoroacetyl azidothiophenes are synthesised from the corresponding 2,3-dibromo, 3,4-dibromo, and 2,5-dibromo thiophene via a one-pot procedure, by stepwise sequential halogen-lithium exchange and subsequent reaction of the produced lithium-bromothienyls with $N, N^{\prime}$-dimethyl acetamide or $N, N^{\prime}$-diethyl-2,2,2trifluoroacetamide. This step results in the formation of bromothiophene intermediates bearing the lithio 'protected' carbonyl groups I, which are then allowed to undergo further halogenlithium exchange and reaction with tosyl azide II. The final treatment of the resulting dilithium salts with diluted hydrochloric acid and then the usual treatment with tetrasodium pyrophosphate produces the appropriate acetyl $\left(\mathrm{R}=\mathrm{CH}_{3}\right)$ and trifluoroacetyl $\left(\mathrm{R}=\mathrm{CF}_{3}\right)$ azidothiophenes $\mathbf{A}$ in reasonable yields $(10-60 \%) .{ }^{17 b}$ (see Scheme 3 )

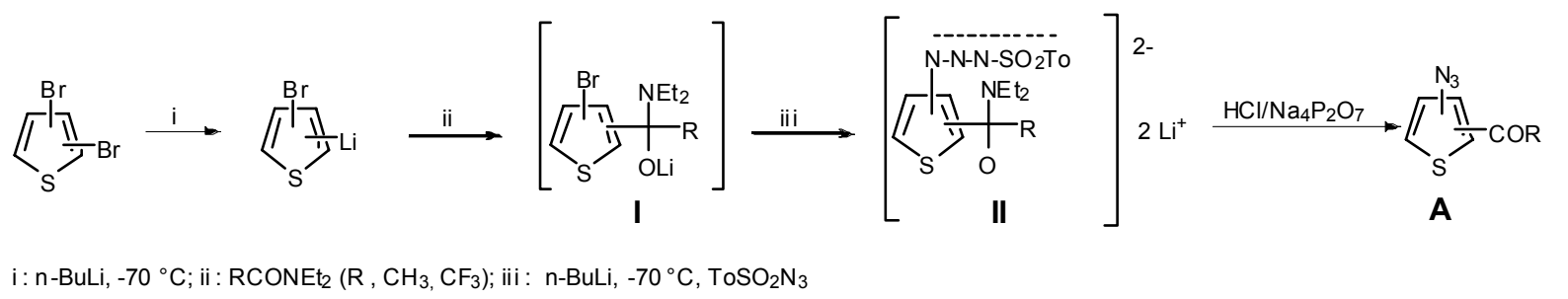

\section{Scheme 3}

This forced procedure has proven to be useful in the preparation of ortho- and para-like substituted oxothienyl azides, whose thermal stabilities depend on the electrical effects exerted by the carbonyl substituent on the azido groups differently positioned on the ring. The thermochemical behaviour of these novel heteroaryl azides prompted us to study the versatility of the 'azido transfer' protocol by extending it to a series of azidoazole compounds (namely: 1,3thiazole, benzothiazole, $N$-methylimidazole, and $N$-methylpyrazole), some of which were previously unknown. In previous studies, the known chemistry of azidoazoles has been focused on thermal ring-cleavage ${ }^{18}$ and on azido-tetrazole isomerism, which have been investigated from experimental ${ }^{19}$ and theoretical viewpoints. ${ }^{20}$

Previously reported simple azidoazoles were generally obtained by the diazotisation of the corresponding available 2-aminoazoles, followed by reaction of the azido ion with the resulting diazonium compounds; ${ }^{21}$ however, this route is unsuitable for the preparation of simple 5azidoazoles. By the lithium 'azido transfer' protocol, simple 2- and 5-azidoazoles were obtained following the protocol illustrated in Schemes 4 and $5^{22}$. The 2-lithium derivatives of $\mathrm{N}$ methylimidazole, ${ }^{23}$ 1,3-thiazole, ${ }^{24}$ benzo-1,3-thiazol, ${ }^{25}$ and $N$-methylpyrazole, ${ }^{26}$ prepared according to the procedures described, ${ }^{27}$ react quantitatively with tosyl azide, resulting in solid azole-2-tosyltriazenyl lithium salts. Final treatment with tetrasodium pyrophosphate produces the appropriate 2-azidoazoles in fairly good yields (63-81\%). 


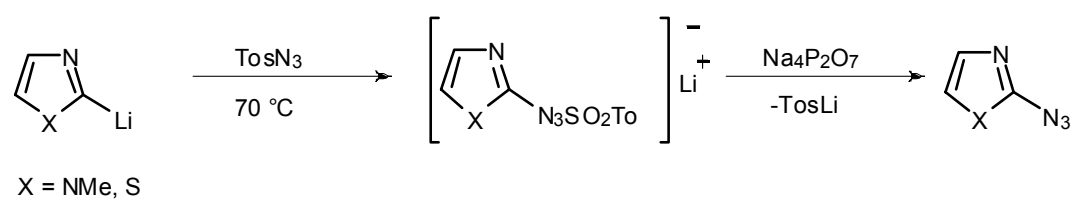

\section{Scheme 4}

Otherwise, the lithiation at the 5-position is only possible when the 2-position is blocked. The simple 5-azido azoles are thus prepared following a two-stage organolithium route, in which the trimethylsilyl group is used as a blocking group for the readily lithiated 2-position, for example, by reacting 2-lithio- $N$-methyl-imidazole and 2-lithio-1,3-thiazole with (trimethylsilyl)chloride. (Scheme 5). The resulting 2-silylated azoles undergo a second lithiation process at the 5-position, and then by reaction with tosyl azide, they form the corresponding 2(trimethylsilyl)-5-triazene lithium salts. Finally, the target 5-azidoazole species are recovered after the usual fragmentation of the corresponding triazene lithium salts - by desilylation of 5azido-2-(trimethylsilyl)-azoles in tetrahydrofuran (THF) at $-5^{\circ} \mathrm{C}$ using tetrabutylammonium fluoride $/ \mathrm{SiO}_{2}$. This latter approach is characterised by short reaction times $(3-4 \mathrm{~h})$ and facile work-ups. $^{28}$ (Scheme 5)

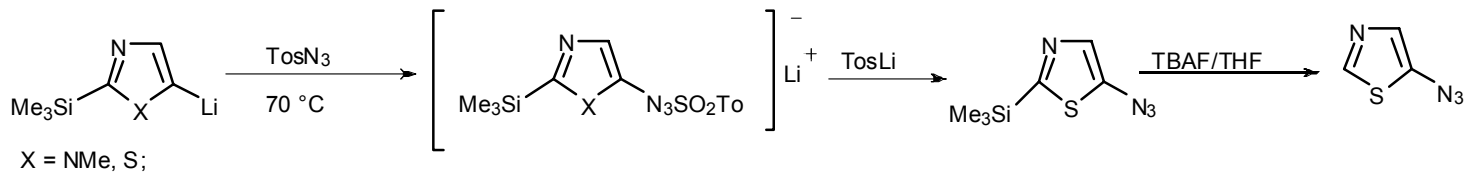

\section{Scheme 5}

The 'azido transfer' synthetic protocol offers virtually limitless opportunities for the synthesis of azides derived from heteroaromatic systems containing one or more heteroatoms that can undergo regioselective lithiation. This opinion is confirmed by the series of azidoazole compounds prepared up to now by processes that are essentially based on the 'azido transfer' protocol (see Scheme 6).

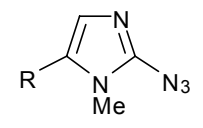

$\mathrm{R}: \mathrm{H} ; \quad(75 \%)$

R: SiMe; $(70 \%)$

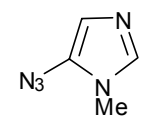

$(10 \%)$

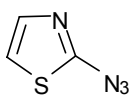

$(70 \%)$

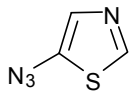

$(56 \%)$

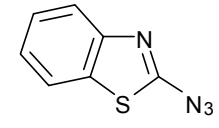

$(72 \%)$

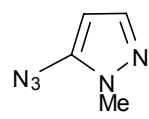

$(63 \%)$

\section{Scheme 6}

The chemistry of 2-azidoazoles - in Scheme 7 where (d) is a nitrogen atom and (a) a heteroatom (i.e., S, NR) - appears to be more complex than that of one-heteroatom 2- 
azidoheteroaryls, which, however, generally undergo thermal ring cleavage into 4-cyano 1,3heterodienes $\mathbf{A}(\mathrm{a}=\mathrm{S}, \mathrm{NMe} ; \mathrm{d}=\mathrm{N})$, despite the possible equilibrium between the azido linear form and the isomeric tetrazole ${ }^{[18]}$.

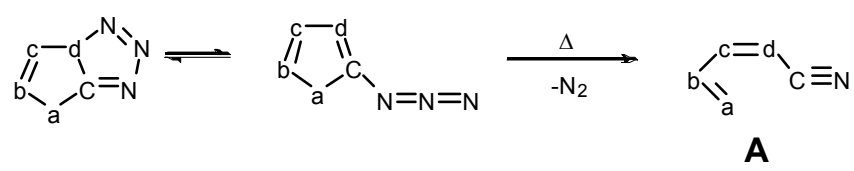

\section{Scheme 7}

The 5-position in the case of azidoazoles is comparable to the 2-position on one-heteroatom heteroaryl systems, such that ring cleavage may occur. (Scheme 7) In practice, 5-azido- $N$ methylpyrazole $(\mathrm{a}=\mathrm{NMe}, \mathrm{b}=\mathrm{N}), 5$-azido-1,3-thiazole $(\mathrm{a}=\mathrm{S}, \mathrm{c}=\mathrm{N})$, and 5-azido- $N$ methylimidazole $(\mathrm{a}=\mathrm{NMe}, \mathrm{c}=\mathrm{N})$ were found to be less stable than 2-azido- $N$-methylimidazole $(\mathrm{a}=\mathrm{NMe} ; \mathrm{d}=\mathrm{N})$ and 2-azido-1,3-thiazole $(\mathrm{a}=\mathrm{S} ; \mathrm{d}=\mathrm{N})$, which are probably stabilised by conjugation with the $s p^{2}$ nitrogen atom (c), and also more unstable than 2-trimethylsilyl-5-azido$N$-methylimidazole, which is slightly stabilised by the para-like 2-trimethylsilyl substituent. ${ }^{22}$ Both the 2-azido and 3-azidoheteroaryls will be regarded as serious candidates to develop different synthetic strategies, some of which are discussed in Chapter 2.2.

\subsection{General reactivity of heteroaryl azides}

The diverse, well-known properties of the azido group - as fast access to an enormous variety of nitrogen-containing derivatives - and the typical reactivity of the nitrene species derived from its dissociation, have allowed us to raise new questions about the fate of these heteroaryl azides. We were pleased to find that the 3-azidoheteroaryls undergo conventional thermal dissociation reactions similar to those of phenylnitrenes, if a nitrene is generated at the 3position, ${ }^{29}$ whereas the 2-azidoheteroaryls usually undergo unimolecular concerted loss of nitrogen and cleavage of the ring with the formation of synthetically useful 4-cyano 1,3heterodiene 'building blocks'. These in turn undergo Diels-Alder type cycloaddition to sixmembered heterocycles in the presence of appropriate dienophiles. ${ }^{30}$ (see $\mathbf{v}$ in Scheme 8)

In this article, we discuss both the synthetic aspects of heteroaryl azides, and the mechanisms involved in their classical or peculiar thermal and chemical reactions (see reactions i-iv in Scheme 8).

i) amines upon reduction of the azido group;

ii) nitrenes upon thermal reaction and dissociation of the azido group;

iii) nitrenium ions upon reaction with a Lewis acid and dissociation of the azido group;

iv) 1,3-dipolar cycloaddition (1,3-DC) in the presence of terminal or disubstituted alkenes and alkynes. 


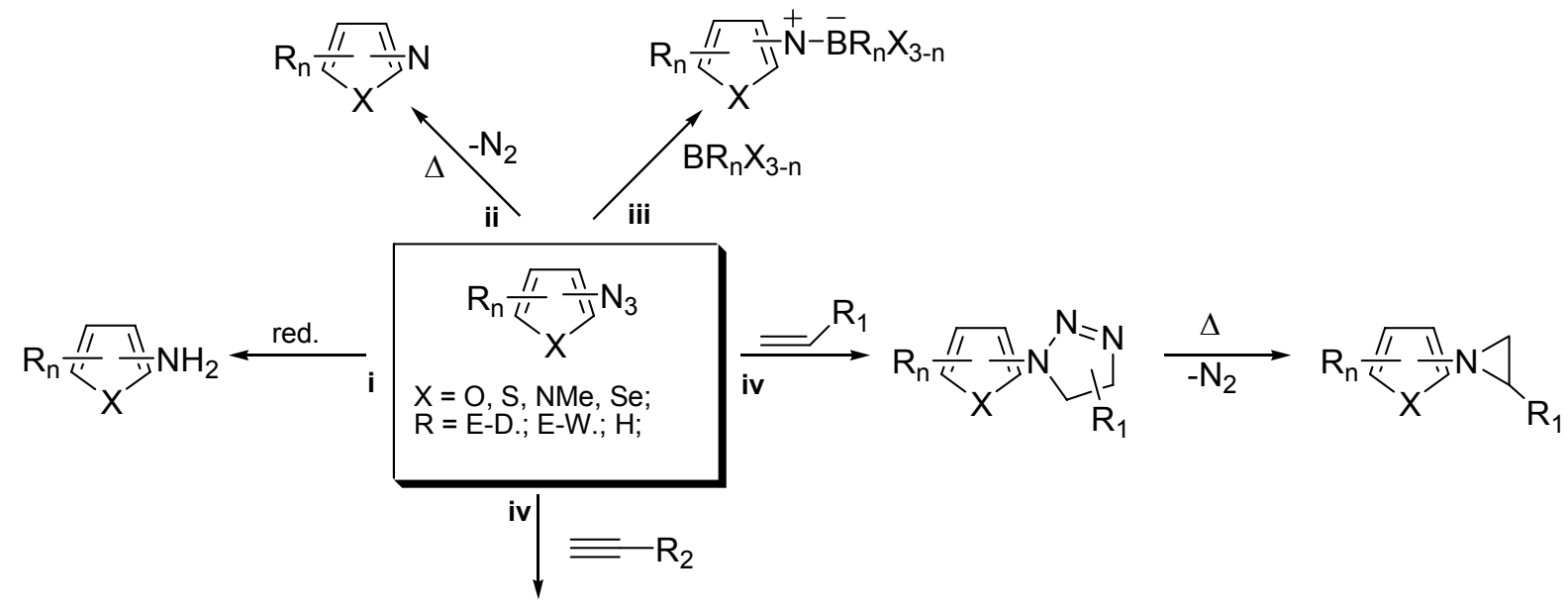

$$
\begin{aligned}
& R_{n} \frac{\sqrt{1}}{x^{\prime \prime}} \frac{1}{y=} N_{R_{2}}^{N=}
\end{aligned}
$$

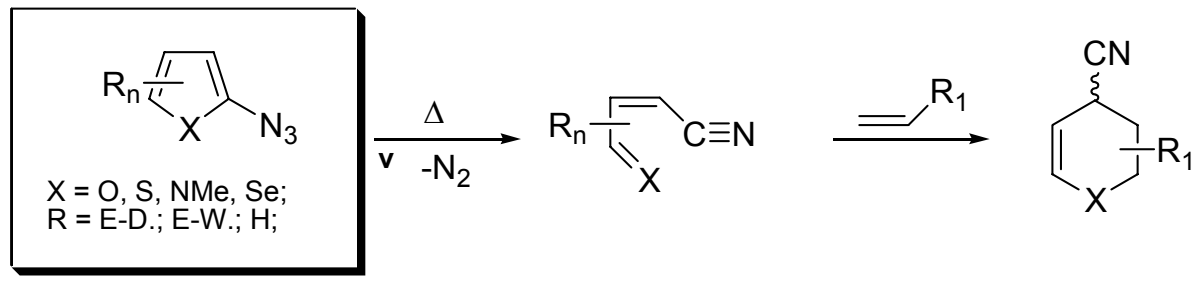

\section{Scheme 8}

\subsection{Heteroaryl amines by the reduction of the azido group}

Organic azides can be easily converted into amines by most of the well-known, conventional methods; ${ }^{31 \mathrm{a}}$ however, the easier reduction process, which is the base-catalyzed reaction with hydrogen sulfide in methanol, appears to be less detrimental to heteroaryl rings, while the byproduct, sulphur, is easily removed by filtration. ${ }^{17 \mathrm{~b}, 31 \mathrm{~b}}$ (see Scheme 9)

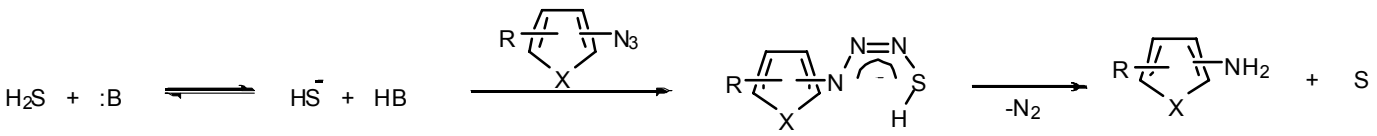

\section{Scheme 9}

For example, the reduction of the six $o$-azidobithienyl isomers using hydrogen sulfide in methanol gives the appropriate $o$-aminobithienyl isomers in an almost quantitative yield (i.e., 87-95\%), the only exception being the rather unstable 2-azido-3,2'-bithienyl (71\%). (Scheme 10) 


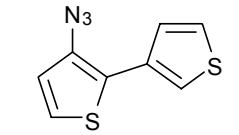

3-Azid o-2,3'-b ithien yl(73\%)

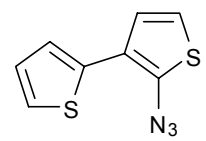

2-Azido-3,2'-bithienyl(33\%)

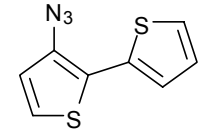

3-Azid o-2,2'-bi thien yl(72\%)

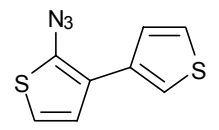

2-Azido-3,3'-bithienyl(41\%)

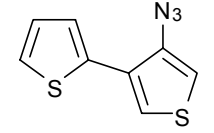

4-Azido-3,2'-bithienyl(75\% )

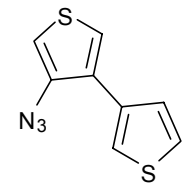

4-Azido-3,3'-bith ienyl(77\%)

\section{Scheme 10}

Since free aminothiophenes have not been previously prepared, the overall 'azido transfer' and its reduction pathways can be considered as a unique and convenient general route for the direct transformation of lithium thiophenes or other similar lithium heteroaryls into amino heteroaryls. $^{32}$

\subsection{Thermal dissociation of heteroaryl azides}

As for the reactions involving pseudo 'nitrene' intermediates, the peculiar low-energy fragmentation and the ring-cleavage process experimentally observed for the simple 2azidoheteroaryls $^{15,33,30 \mathrm{a}}$ follow the same rule when 2-azidobenzofuran, 2-azido- $N$-methylindole, and 2-azidobenzothiophene (2-BTA) are studied. Mild thermal decomposition of these compounds in an inert solvent produces mainly dimeric products that are diagnostic for determining both the reaction times and the different reactivities of the cleaved intermediates (Scheme 11).

A remarkably high reactivity is shown by the incoming ring-opened $o$-quinoidal enone $\mathbf{C}, \mathrm{X}$ $=\mathrm{O} ; 2$-(6-oxo-2,4-cyclohexadienyliden)acetonitrile (Scheme 11) with the ene part of the furan ring of the starting 2-azidobenzofuran, which yields the final tetracyclic azide $\mathbf{C 1}$ as the major product. The operation of this type of [4+2]-cycloaddition is probably due to the greater instability of the 2-azidobenzofuran $\left(15 \mathrm{~min}\right.$ half-life at $\left.60^{\circ} \mathrm{C}\right)$, and the presence of a good dienophile among the three azides under investigation. ${ }^{16,34}$ 

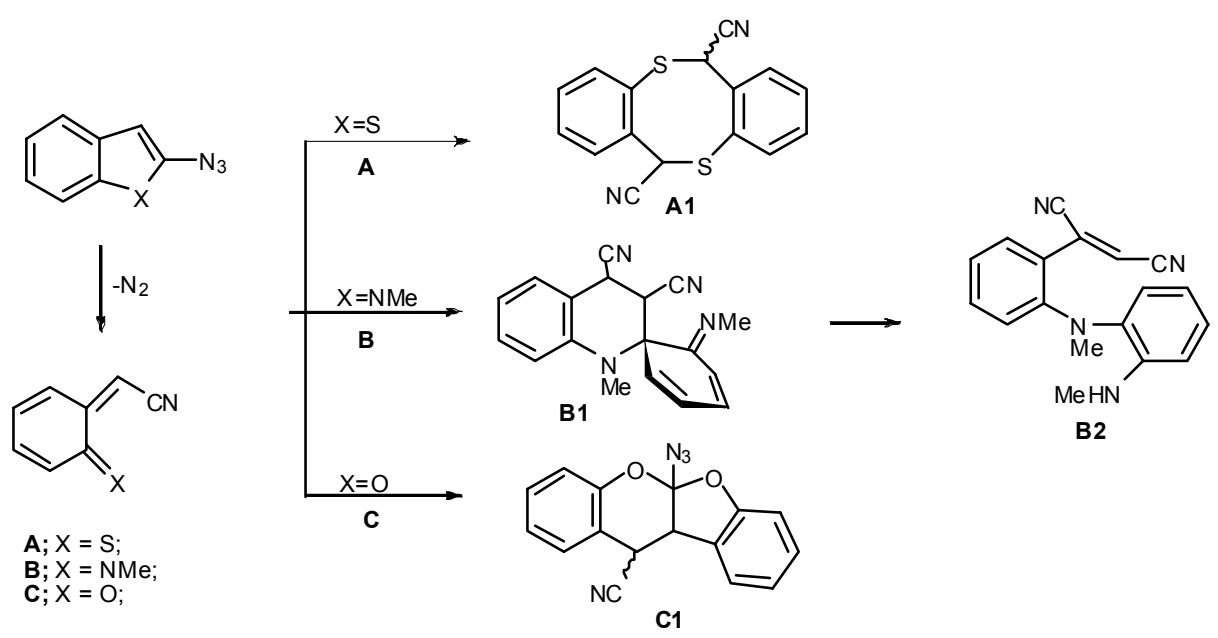

\section{Scheme 11}

A [4+2]-cyclodimerisation of the $o$-quinone methyneimine - namely 2-[6-(methylimino)2,4-cyclohexadienyliden]acetonitrile $(\mathbf{B}, X=\mathrm{NMe}$; Scheme 11) obtained by thermal dissociation of the 2-azido- $\mathrm{N}$-methylindole $\left(45 \mathrm{~min}\right.$ at $\left.60^{\circ} \mathrm{C}\right)$ - gives mainly the (Z)-2-\{2-[methyl-2(methylamino)anilino]phenyl\}-2-butenedinitrile B2, whose structure was confirmed by X-ray diffraction. The cycloadduct $\mathbf{B} 2$ is the rearrangement product of the former spiro-cycloadduct B1, produced via [4+2]-cycloaddition-dimerisation of the $o$-quinone methyneimine $\mathbf{B}$. On the other hand, the thermal dissociation $\left(10 \mathrm{~h}\right.$ at $\left.60^{\circ} \mathrm{C}\right)$ of 2-BTA yields a mixture of $(E)$ and $(Z)$ $6 H, 12 H$-dibenzo[ $b, f]$ dithiocine-6,12-dicarbonitrile A1 (Scheme 11).

A mechanistic proposal can be postulated for the cyclodimerisation of the ring cleaved intermediate thioxo dienylidene A (Scheme 12) that would produce dithiocine-6,12dicarbonitrile A1 via an 1,4-head-tail self-addition of the mesomeric form of its thioxo dienylidene component $\mathbf{A}$.

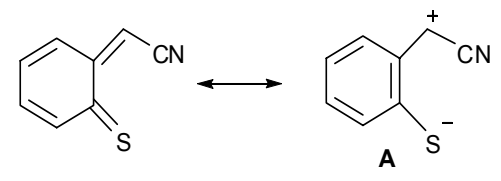

\section{Scheme 12}

Nevertheless, the three $o$-quinone intermediates $\mathbf{A}, \mathbf{B}$, and $\mathbf{C}$ are useful building-blocks for $[4+2]$-cycloadditions when the thermal dissociations of the starting 2-azides are performed in the presence of dienophiles alkenes.

Kinetic studies - based on both a decrease in the intensity of the neat IR band of the azido group (observed at about $2100-2150 \mathrm{~cm}^{-1}$ ) and an increase in pressure resulting from the formation of molecular nitrogen, - have revealed that the first-order rate constant obtained for 
the unimolecular dissociation of the azido group at $80^{\circ} \mathrm{C}$ in $p$-chlorotoluene for 3-azidothiophene $\left(k_{3}=1.35 \cdot 10^{-6} \mathrm{~s}^{-1}\right)$ was $k_{2} / k_{3}=2430$ times slower than that observed for 2-azidothiophene $\left(k_{2}=\right.$ $\left.3.16 \cdot 10^{-3} \mathrm{~s}^{-1}\right)$. $^{33}$ In the same way, the rate constant for the dissociation of 3 -azidoselenophene $\left(k_{3}\right.$ $\left.=2.50 \cdot 10^{-6} \mathrm{~s}^{-1}\right)$ is $k_{2} / k_{3}=2480$ times slower than that of the 2 -azidoselenophene $\left(k_{2}=6.21 \cdot 10^{-3} \mathrm{~s}^{-}\right.$ $\left.{ }^{1}\right)^{12}$

The great differences in the kinetic values suggest higher mesomeric conjugation in simple 2-azido -thiophene or -selenophene, which favours concerted ring cleavage and the loss of dinitrogen process with respect to the 3-position - and reduces the probability of low-energy mesomeric structures - presumably following the trend of stabilisation of the resulting ring cleaved carbon-heteroatom double bond part of the final 4-cyano 1,3-heterodienes $(\mathrm{C}=\mathrm{O}>$ $\mathrm{C}=\mathrm{NR}>\mathrm{C}=\mathrm{Se} \approx \mathrm{C}=\mathrm{S}){ }^{30 \mathrm{a}}$ (Scheme 13 , path II)

This suggestion is supported by the lower activation energies and the larger negative entropies calculated for the decomposition of the 2-azido thiophene $\left(E_{\mathrm{a}}=22.6 \mathrm{kcal} \mathrm{mol}^{-1} ; S_{\mathrm{a}}=\right.$ $\left.8.2 \mathrm{cal} \mathrm{K}^{-1} \mathrm{~mol}^{-1}\right)^{33}$ and 2-azido selenophene $\left(E_{\mathrm{a}}=21.5 \mathrm{kcal} \mathrm{mol}^{-1} ; S_{\mathrm{a}}=-10.7 \mathrm{cal} \mathrm{K}^{-1} \mathrm{~mol}^{-1}\right)^{12}$ compounds, compared to those obtained for the corresponding 3-azido species $\left(E_{\mathrm{a}}=30.7 \mathrm{kcal}\right.$ $\mathrm{mol}^{-1} ; S_{\mathrm{a}}=-0.7 \mathrm{cal} \mathrm{K}^{-1} \mathrm{~mol}^{-1}$, and $E_{\mathrm{a}}=30.4 \mathrm{kcal} \mathrm{mol}^{-1} ; S_{\mathrm{a}}=-0.9 \mathrm{cal} \mathrm{K}^{-1} \mathrm{~mol}^{-1}$, respectively). It is important to note that, since the ring cleavage represented by path II (see Scheme 13) involves low energy barriers, the possibility of a thermodynamically favourable formation of $\mathrm{C}=\mathrm{X}$ double and $\mathrm{CN}$ triple bond exists.

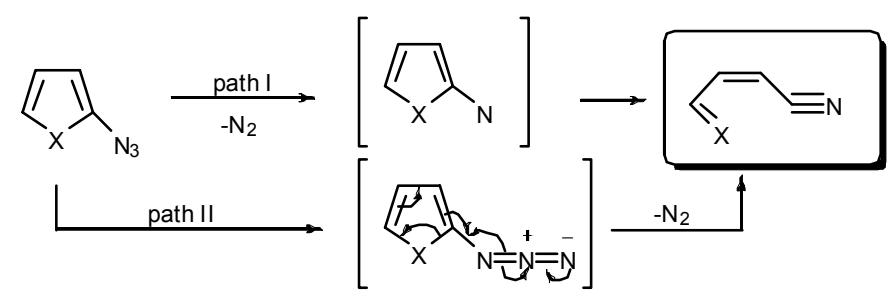

\section{Scheme 13}

A molecular modelling of the two processes (paths I and II illustrated in Scheme 13) was performed, including CI-Singles energy- and geometry-optimised calculations for both 2-azidothiophene and -selenophene structures. The calculations provide a reasonable description of the process, which proceeds via the opened 1,3-heterodiene structure in cisoid conformation, via a

concerted unimolecular mechanism instead of the intermediate stage of a 'free nitrene'. ${ }^{30 \mathrm{~b}}$ The optimised initial azides and final ring cleaved geometries by MP2/6-31G*, with frequencies at $\mathrm{HF} / 6-31 \mathrm{G}^{*}$, provide evidence for a greater stabilisation of the 4-oxobut-2-enenitrile with respect to the 4-thioxobut-2-enenitrile, given the respective heats of formation produced by the thermochemical recipes $\mathrm{T} 1\left(-219.3 \mathrm{~kJ} \mathrm{~mol}^{-1}, \mathrm{X}=\mathrm{O}\right.$ and $\left.-153.9 \mathrm{~kJ} \mathrm{~mol}^{-1}, \mathrm{X}=\mathrm{S}\right)$. (Scheme 13) Kinetic studies showed less marked differences $\left(k_{2} / k_{3} \approx 309\right.$ at $\left.80^{\circ} \mathrm{C}\right)$ in the rate constant dissociation of 2-BTA and 3-azidobenzo[b]thiophene (3-BTA) than the corresponding 2- and 3azidothiophenes. These rates are associated with the calculated low activation energies and larger 
negative entropies: $E_{\mathrm{a}}=20.9 \mathrm{kcal} \mathrm{mol}^{-1}, S_{\mathrm{a}}=-16.7 \mathrm{cal} \mathrm{K}^{-1} \mathrm{~mol}^{-1}$ for the 2-BTA and $E_{\mathrm{a}}=24.4 \mathrm{kcal}$ $\mathrm{mol}^{-1}$, and $S_{\mathrm{a}}=-13.6 \mathrm{cal} \mathrm{K}^{-1} \mathrm{~mol}^{-1}$ for the 3-BTA. ${ }^{6 \mathrm{c}}$ It seems that the partial involvement of the 'aromaticity' stabilises $o$-quinoidal transition structures favouring the dissociation of the 2-azido and, to a greater extent, of the 3-azidobenzoheteroayls with comparable energetic values in between. ${ }^{6 \mathrm{~b}}$ The comparable kinetic and thermodynamic data measured for these 2- and 3azidobenzo-fuse systems - far from the values reported for the thermolysis of phenyl azide, 3azido-thiophene, and -selenophene to the corresponding nitrene, but similar to those observed for the conversion of vinyl azide to $2 H$-azirine - suggest that the TD of the 3-BTA mainly occurs with the assistance of a nitrene/azirine mechanism. (Scheme 14)

When the thermolysis of 3-BTA is performed in the dark at $90^{\circ} \mathrm{C}$ in the presence of nucleophilic dialkylamines (dimethylamine or diethylamine), the $N^{3}, N^{3}$ dialkylbenzo $[b]$ thiophene-2,3-diamines $\mathbf{B}$ - whose structures have been confirmed by independent methods - are isolated in fairly good yields (75-78\%), together with small amounts of 3-aminobenzo[b]thiophene (6-10\%). ${ }^{6 c}$ (see Scheme 14$)$

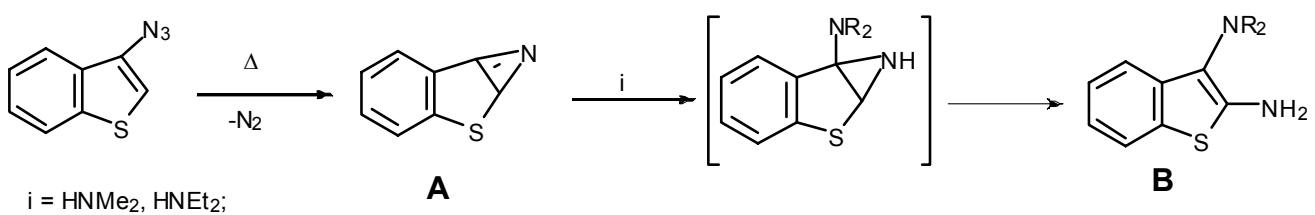

\section{Scheme 14}

Identical $N^{3}, N^{3}$-dialkylbenzo[b]thiophene-2,3-diamines $\mathbf{B}$ are isolated, as well as a small amount of 2-aminobenzo[b]thiophene $(3-5 \%)$, with 2-BTA at $60^{\circ} \mathrm{C}$. This result suggests that the nitrene/azirine ring formation is favoured in only the case 3-BTA, wherein the thermal process in the presence of dialkylamines proceed via attack at the $\mathrm{C}=\mathrm{N}$ double bond of the intermediate azirine $\mathbf{A}$ to give the diamino $\mathbf{B}$ in very good yields (85 - 87\%) (Scheme 14). In addition, the decompositions of 2-BTA and 3-BTA with sulphur-nucleophiles, such as 1-ethyl- and 1-butylthiol, were carried out in the dark at room temperature. In these instances, 3-BTA is converted within a week in $80 \%$ yield to 2-(alkylthio)-3-aminobenzothiophenes A, while the reaction of 2BTA in 1-butylthiol gives the 3-butylsulfanyl-2-aminobenzothiophene $\mathbf{B}$ as the major product (with a 55\% yield), in addition to by-products due to the well known unimolecular concerted 2azidoheteroaryl dissociation and ring-cleavage process. ${ }^{6 c}$ In both cases, a mechanism involving the nitrene/azirine ring appears to be precluded and the outcomes can be attributed to the kinetically favoured vinylazido ylide mesomeric forms. (Scheme 15) 


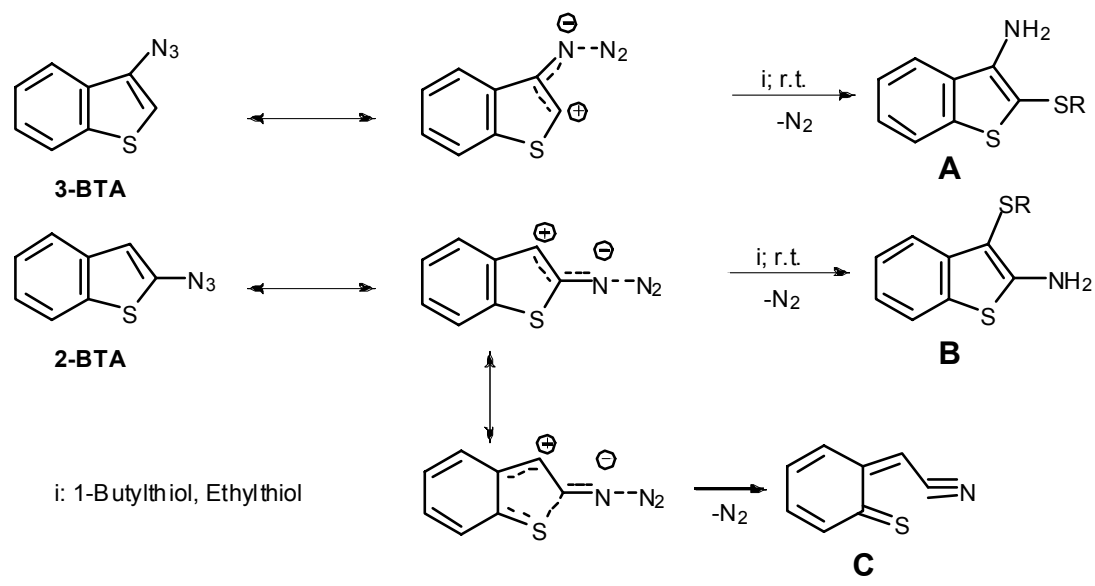

\section{Scheme 15}

Structural effects on the thermal behaviour of few organic azides have been determined by EI mass spectrometry under the same instrumental conditions $(70 \mathrm{eV}, \mathrm{P}, \mathrm{T}$, and $\mathrm{m} / z)$ for a homogeneous class of compounds. ${ }^{35}$ Similarly, the MS spectral data for simple heteroaryl azides, like 2-azido- and 3-azido derivatives of the thiophene and selenophene series, follow a general pattern that reflects the single structural features. ${ }^{12,33}$ As expected, the molecular ions (HetN$\mathrm{N}_{2}{ }^{++}$) of the isomeric 3-azides $(\mathbf{2}, \mathbf{4})$ are greater than those of the 2-azides $(\mathbf{1}, \mathbf{3})$ ' and the primary fragmentation - which correspond to the loss of neutral $\mathrm{N}_{2}(\mathrm{M}-28)^{\circ+}$ with the release of fragments at $\mathrm{C}_{4} \mathrm{H}_{3} \mathrm{NS}^{\cdot+}$ and at $\mathrm{C}_{4} \mathrm{H}_{3} \mathrm{NSe}^{\cdot+} m / z$ from the molecular ion $\left(\mathrm{M}^{\cdot+}\right)-$ are reported in Scheme 16.
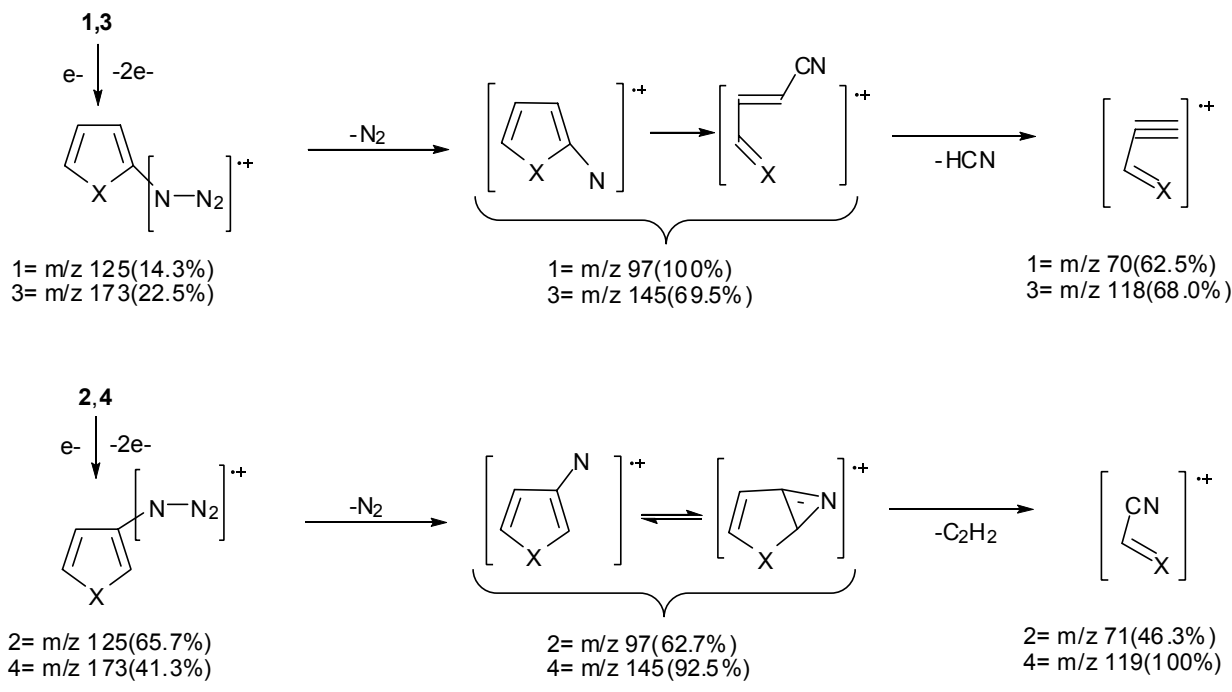

\section{Scheme 16}


The critical energy of the process is equivalent to the dissociation energy of the HetN-N 2 bond plus the ionisation energy $(I)$ of the radical $\mathrm{HetN}^{\circ+}$, since the reverse process is insignificant. The next processes are characterised by diverse fragmentations showing prevalent elimination of hydrogen cyanide $(\mathrm{HCN})$ from the 2-azido $\mathbf{1}, \mathbf{3}$, and of acetylene $\left(\mathrm{C}_{2} \mathrm{H}_{2}\right)$ from the 3-azido 2, 4. Although the collected MS data of simple heteroaryl azides 1-4 are limited, in principle, the correlation between the geometric and structural features of these compounds and the MS results are confirmed.

Since the pioneering work of Smith and Brown in $1951,{ }^{36 \mathrm{a}}$ it is widely known that $o$ azidobiphenyl derivatives can yield carbazole ( $>95 \%$ ) upon thermolysis under 'controlled' conditions in an inert solvent, via a six-electron pericyclic 1,5-cyclisation of a nitrene singlet intermediate. $^{36 \mathrm{~b}}$ (see Scheme 17) Similar thermal decomposition routes have been extensively further studied in the presence of various suitable substituents on the phenyl rings and different solvents to recognise the nitrene singlet/triplet state. ${ }^{37 \mathrm{a}-\mathrm{d}}$

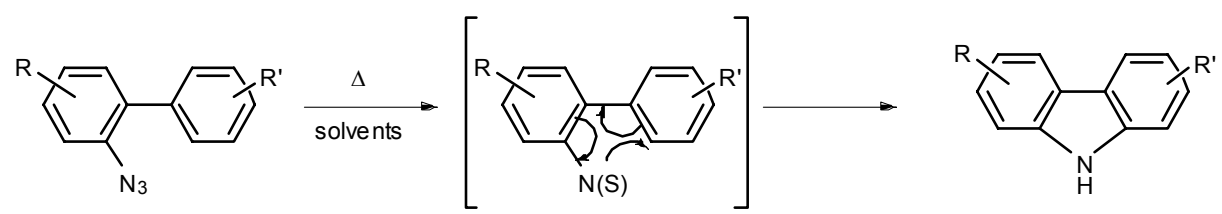

\section{Scheme 17}

It is well known that the activation energy values for the thermolyses of $\alpha$, $\beta$-unsaturated $o$ substituted phenyl azides are generally low (between 22 and $27 \mathrm{kcal} \mathrm{mol}^{-1}$ ), and have large negative entropic values; however, in the case of the $o$-azidobiphenyls the dissociation appears to proceed with feeble mesomeric assistance, with activation energy values ranging between 32.5 and $40.6 \mathrm{kcal} \mathrm{mol}^{-1}$, together with small negative entropies. ${ }^{37 a}$ Interestingly, in the course of a recent calorimetric investigation of the hazardous properties of some aryl azides and diazides, we have observed that the thermal decomposition rates $\left(\mathrm{TD}, t_{1 / 2}\right)$ at $160{ }^{\circ} \mathrm{C}$ of the 4 -azidobiphenyl were three times slower than that of the 2-azidobiphenyl, while the respective activation energies were 37.8 and $23.7 \mathrm{kcal} \mathrm{mol}^{-1}$; however, the formation of carbazole was detected at a yield of only about $36 \%$ by C80-FTIR analysis. In this case, the exothermic TD of 2-azidobiphenyl mainly proceeds via a pericyclic intramolecular 1,5-cyclisation that is characterised by a lower activation energy, higher negative entropic value, and rate enhancement with respect to the isomeric 4-azidobiphenyl. ${ }^{2 a}$

One would like to think that the thermal decomposition in solution of the six $o$ azidobithienyl compounds - shown in Scheme 10 - would be a facile source of isomeric dithienopyrroles. ${ }^{38}$ Conversely, the 2-azido-3,3'- and 2-azido-3,2'-bithienyl thermal behaviours are considerably different from those of 3-azido-2,2'- and 3-azido-2,3'-bithienyl, and 4-azido3,2'- and 4-azido-3,3'-bithienyl. Due to their high instability, the fragmentation of the 2-azido isomers occurs, extruding nitrogen at room temperature, without any sign of 1,5-cyclisation 
products. In the case of the 2-azido-3,3'-bithienyl, the process results in a mixture of isomeric $m$ ditiine compounds produced via [4+2]-self-cycloaddition of the enethione $\mathbf{A}$ - which is diagnostic of the ring cleavage (see Scheme 18) — whereas the fragmentation of the 2-azido3,2'-bithienyl yields only a resinous intractable material.

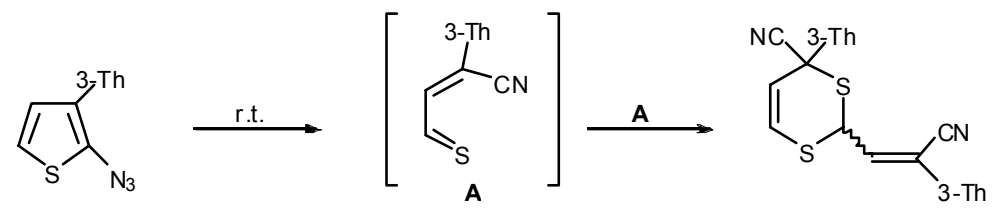

\section{Scheme 18}

Successful fragmentation of the 3-azido-2,2'-, and 3-azido-2,3'-bithienyl, and 3-azido-2cyclohexenylthiophene compounds in chlorobenzene at $132^{\circ} \mathrm{C}$ gives the target $4 H$-dithieno[3,2$\left.b ; 2^{\prime}, 3^{\prime} d\right]$ pyrrole, $\quad 4 H$-dithieno[3,2- $\left.b ; 2^{\prime}, 3^{\prime} d\right]$ pyrrole, and 5,6,7,8-tetrahydro-4H-thieno[3,2b]indole in very good yields (about $87-88 \%$ ). (Scheme 19)

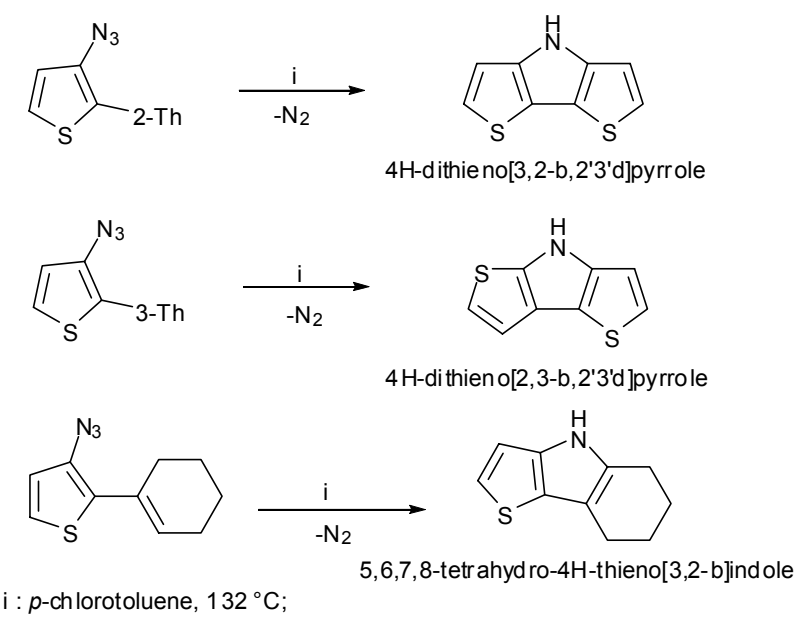

\section{Scheme 19}

An interesting question arises in the case of $o$-azidobithienyls, such as 4-azido-3,2'- and, 4azido-3,3'-bithienyl, and 4-azido-3-cyclohexenyl-thiophene. These compounds do not yield the expected tricyclic thienopyrroles - not even at higher temperatures $\left(178{ }^{\circ} \mathrm{C}\right)$ - while the starting material or, when forced, polymeric materials do. These 'failures' are imputable to the lack of low-energy structures to makes pericyclic 1,5-cyclisation pathways for the reactions of these isomers. ${ }^{38}$ 


\subsection{Nitrenium ions via azido fragmentation with boron derivatives}

The chemical decomposition of organic azides in strong protic medium has been studied from both synthetic and theoretical points of view; $;^{3 a, 39,40}$ however, the undervalued decomposition of heteroaryl azides in the presence of hard Lewis acids also requires focused attention.

Consequently, the room temperature treatment of the $o$-azidobiphenyl with $\mathrm{BF}_{3}$ gas gives carbazole (up 91\%), via intramolecular electrophilic 1,5-cyclisation of a nitrenium-borontrihalide complex, which is formed by ready boron-attack and nitrogen extrusion from the azido group. ${ }^{41}$ (Scheme 20)

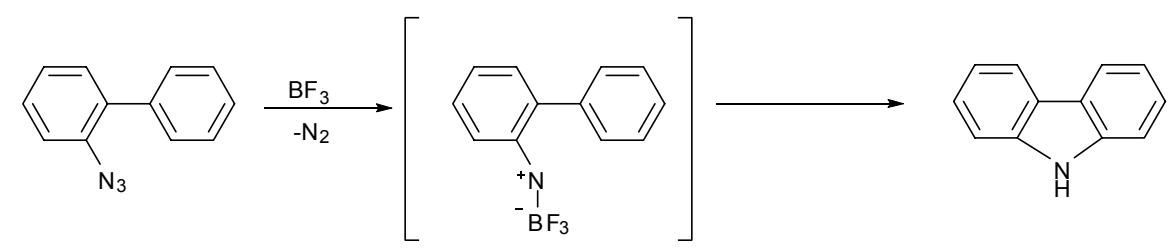

\section{Scheme 20}

This kind of result mimics those of the thermal decomposition of the 2-azidobiphenyl to carbazole via nitrene intermediate. ${ }^{37,42}$ On this basis, a similar concerted mechanism (via $\pi 4$ system) involving the singlet nitrenium ion empty orbital $(\pi 0)$ can be suggested in the instance of the $\mathrm{BF}_{3}$-promoted decomposition of the 2-azidobiphenyl to carbazole.

Conversely, when the reaction of the same 2-azidobiphenyl is carried out with a lesser hard acid, such as $\mathrm{PhBCl}_{2}$, a mixture of $N$-phenylbiphenyl-2-amine $\mathbf{B}$ and carbazole $\mathbf{A}$ in a ratio $(8: 2)$ is obtained. (Scheme 21) The outcome primarily depends on the migratory aptitude of the substituent linked to the initial boron atom. ${ }^{43}$

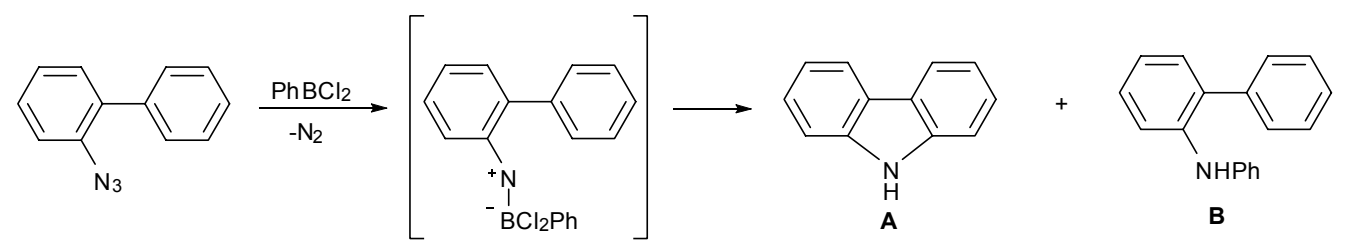

\section{Scheme 21}

Similarly, the 2-azidobiphenyl reacts, in the presence of various nucleophilic solvents, with boron trifluoride-diethyl ether $\left(\mathrm{BF}_{3} \cdot \mathrm{OEt}_{2}\right)$, only at temperatures over $60^{\circ} \mathrm{C}$. The advantage of this reaction is that an exact amount of Lewis acid is employed to form carbazole and/or 2(arylamino)biphenyl via competitive intra- or inter-molecular reactions. It should be noted that the complete decomposition of an aryl azide generally occurs in a few minutes at room temperature with boron trifluoride gas, but essentially does not proceed in the presence of 
$\mathrm{BF}_{3} \cdot \mathrm{OEt}_{2}$. Evidently the ethereal complex largely hinders the formation of the azide- $\mathrm{BF}_{3}$ complex, which, however, could be achieved when the temperature is increased to $60-65^{\circ} \mathrm{C}$. Under these conditions, the phenyl azide- $\mathrm{BF}_{3}$ complex loses molecular nitrogen in a ratedetermining step to give a phenylnitrenium- $\mathrm{BF}_{3}$ complex (see Scheme 22; $\mathrm{Ph}=\mathrm{H}, \mathrm{X}=\mathrm{F}$ ). The rate constant of the decomposition was found to be greater in benzene than in toluene or $m$ xylene. A mechanism involving electrophile nitrenium ions attack is discerned on the basis of the nucleophilicity of the solvent used, while the possibility of an $\mathrm{S}_{\mathrm{N}} 2$ process involving nucleophilic attack by aromatic solvent on the initial azide- $\mathrm{BF}_{3}$ complex is ruled out. Then, the phenylnitrenium- $\mathrm{BF}_{3}$ complex in benzene, toluene, ethylbenzene, fluorobenzene, $m$-xylene, or anisole preferentially forms diphenylamines (Scheme 22), thereby following a pseudo first-order decomposition pathway, whose linear plot correlation between the rate constant for the formation of $p$-substituted diphenylamines $\left(\log F_{\mathrm{p}}\right)$ and the substituent constant $\sigma_{\mathrm{p}}$ indicates a greater electrophilic power than that of the arylnitrenium ion generated by protic decomposition of the azido group ( $\rho$ value -6.69 and -4.4 respectively). ${ }^{40 \mathrm{~b}}$

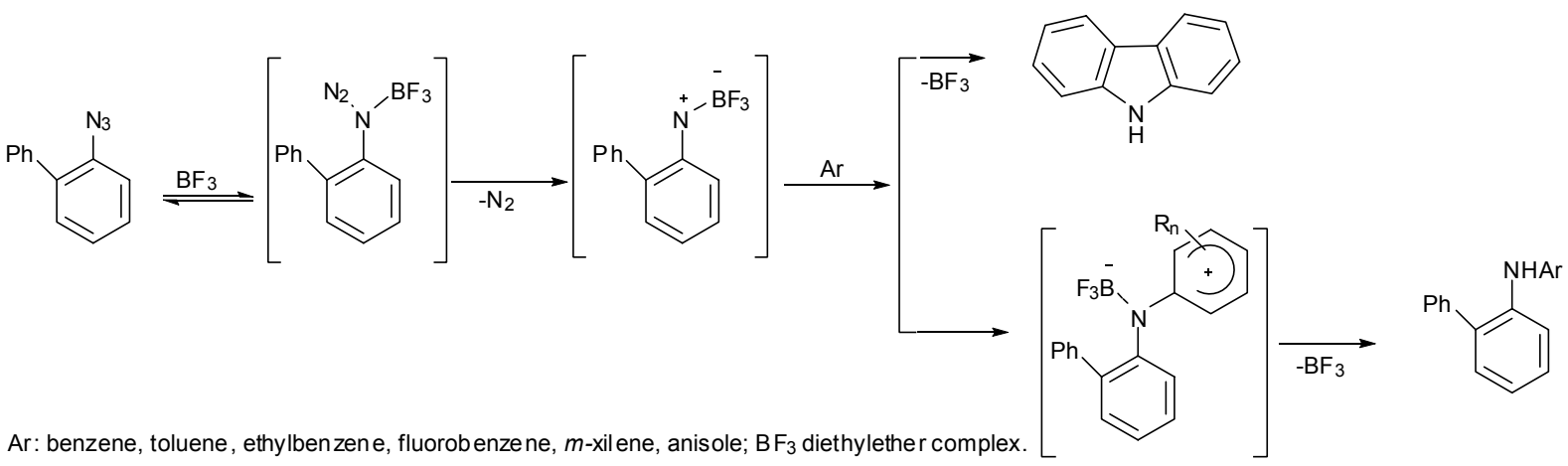

\section{Scheme 22}

Competitive reactions - using a series of $o-, m-, p-\mathrm{CH}_{3}, m-, p-\mathrm{Cl}, m-$, and $p-\mathrm{NO}_{2}$-phenyl azides in the presence of nucleophile methylbenzenes with $\mathrm{BF}_{3} \cdot \mathrm{OEt}_{2}$ - were carried out in an attempt to discriminate the different nitrenium-singlet or -triplet electronic states in the process. ${ }^{376,42}$ In these instances, we observed that the $\mathrm{N}$-substitution products increase at the expense of the diradical $\mathrm{C}-\mathrm{H}$ insertion ones, as the nucleophilicity of the solvent increases. However, the ratio of the $\mathrm{C}-\mathrm{H}$ insertion increases at the expense of the $\mathrm{N}$-substitution by increasing the electron-donating power of the substituent on the phenyl azide ring. As performed, the tests indicate that the conjugate electron-donating group effects on the intermediate nitrenium ion reflect the persistence of its lifetime, and consequently, the intersystem crossing to the triplet state. $^{43}$

Different outcomes are obtained with Lewis acids of different acidic strengths - which is due to an increase in the mobility of the boron substituent. For example when the reaction of the $o$-azidobiphenyl depicted on Scheme 23 is performed with $\mathrm{PhBCl}_{2}$ or $\mathrm{Ph}_{2} \mathrm{BCl}$ - instead of $\mathrm{BF}_{3}$ 
or $\mathrm{BCl}_{3}$ - there is a decrease of carbazole in favour of the secondary biphenyl amines, with respective ratios of $2.0 / 8.0,0.2 / 9.8$ and $10 / 0 \approx 10 / 0 .^{44}$

In principle, it could be possible that the reactions of the six $o$-azidobithienyl isomers those reported in Scheme 11, as well as 4-azido-3-cyclohexen-1-ylthiophene - with boranestype $\mathrm{R}_{\mathrm{n}} \mathrm{BX}_{3-\mathrm{n}}$ would have similar outcomes; however, in these instances, the greater nucleophile character of the vicinal thiophene ring (or the vinyl component) prevails. For example, these compounds react in the presence of $\mathrm{PhBCl}_{2}$ to give fused heterocycles containing, at once, nitrogen and boron atoms via nitrenium-boron complex intermediates. (Scheme 23)

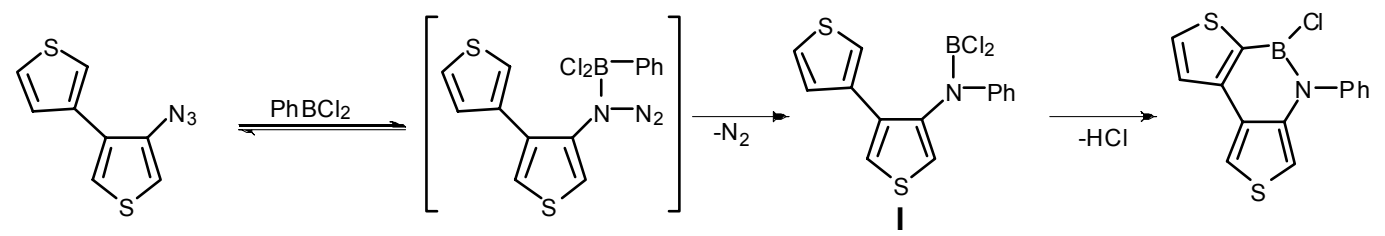

\section{Scheme 23}

The elimination of molecular nitrogen - subsequent to the attack of the $\mathrm{PhBCl}_{2}$ to the azido group - occurs concurrently with the 1,2-shift of the phenyl ring affording dichloroborylamine intermediates $\mathbf{I}$, whose boron atom is still a strong electrophile. These intermediates finally yield tricyclic chloro[1,2]azaborinines, such as 1,2-dihydro-1-phenyl-2-chlorodithieno[b]-, -2chlorodithieno[c]-, and 6-chloro-5-phenylhexahydrodibenzo[c,e]-[1,2]azaborinine, by intramolecular 1,6-cyclisation via aromatic electrophilic substitutions on the vicinal thiophene ring (or the vinyl component). ${ }^{44,45}$ (Scheme 23 )

The resulting moisture-sensitive chloro[1,2] azaborinines - rather than attempt to separate them from the reaction medium - are easily converted into stable 5,5'-oxybis-4-phenyl-4,5dihydrothieno-[1,2]azaborinines A or 5- methoxy-4-phenyl-4,5-dihydrothieno-[1,2] azaboronines B by spontaneous hydrolysis or methanolysis, respectively, with water or methanol. ${ }^{44}$ (Scheme 24)

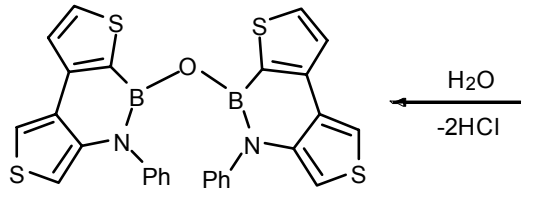

A

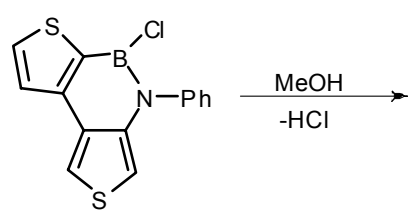<smiles>COB1c2sccc2C2=C[B]C=C2N1c1ccccc1</smiles>

\section{Scheme 24}

The fate of the reactions of the $\mathrm{PhBCl}_{2}$ and $o$-aminobithienyls - namely, 3-amino-2,2'bithienyl, 4-amino-3,3'-bithienyl and 3-amino-2,3'-bithienyl - compared with that of corresponding $o$-azidobithienyls, offers another mechanistic point of view. In these instances, the 
amino-nitrogen attack by the boron atom, followed by the loss of $\mathrm{HCl}$, generates the new intermediates $N$-phenylchloroborylamine I, wherein the phenyl 1,2-shift is precluded. Subsequently, the intermediates $I$ give rise to the corresponding $N$ phenylborazarobenzodithiophenes - namely, 5-phenyl-4,5-dihydrodithieno[3,2-c;2,3-e]-, 5phenyl-4,5-dihydrodithieno[3,4-c;3,4-e]-, and 5-phenyl-4,5-dihydrodithieno[3,4-c;2,3-e]$[1,2]$ azaboronine - arising by intramolecular 1,6-cyclisation via boron electrophilic substitution. ${ }^{46}$ (see Scheme 25)
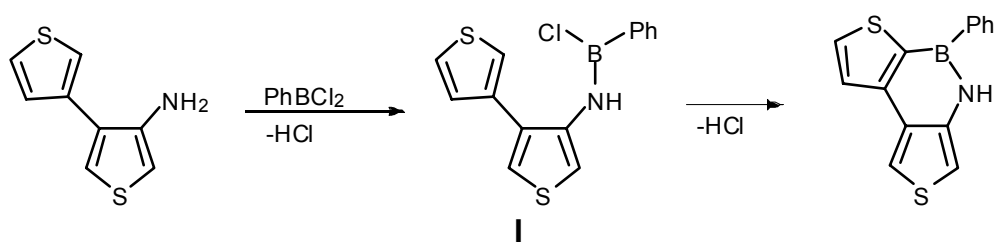

\section{Scheme 25}

\subsection{1,3-dipolar cycloadditions of heteroaryl azides}

Advances in the chemistry of the azidoheteroaryls can be further achieved in the presence of neat dipolarophile alkenes or alkynes, which normally react as solvents/co-reagents to give 1,2,3triazolines or 1,2,3-triazoles regioselectively; via 1,3-dipolar cycloaddition (1,3-DC). But the 1,2,3-triazolines $\mathbf{B}$, which are obtained from 1,3-DCs with both 2-azido and 3-azido substituted heteroaryls, were found to be abnormally unstable compared to those obtained from the aryl azides and smoothly result in the corresponding aziridines $\mathbf{C}$, via quick loss of molecular nitrogen followed by ring-contraction. ${ }^{47}$ (Scheme 26)

Moreover, as already mentioned in the instance of the 2-azidoheteroaryls, these 1,3-DCs are in competition with [4+2]-cycloadditions - Diels-Alder-type - of the heteroaryl ring cleaved products, such as 4-cyano 1,3-heterodienes A (see Scheme 8) and o-quinoidal cyanomethide derivatives. (see Scheme 12) In turn, these building blocks can be trapped in the presence of dienophile alkenes to give 4-cyano six-membered heterocycles, such as pyrans, thiopyrans, chromans, thiochromans, and tetrahydroquinoline A, via [4+2]-cycloaddition. (Scheme 26)

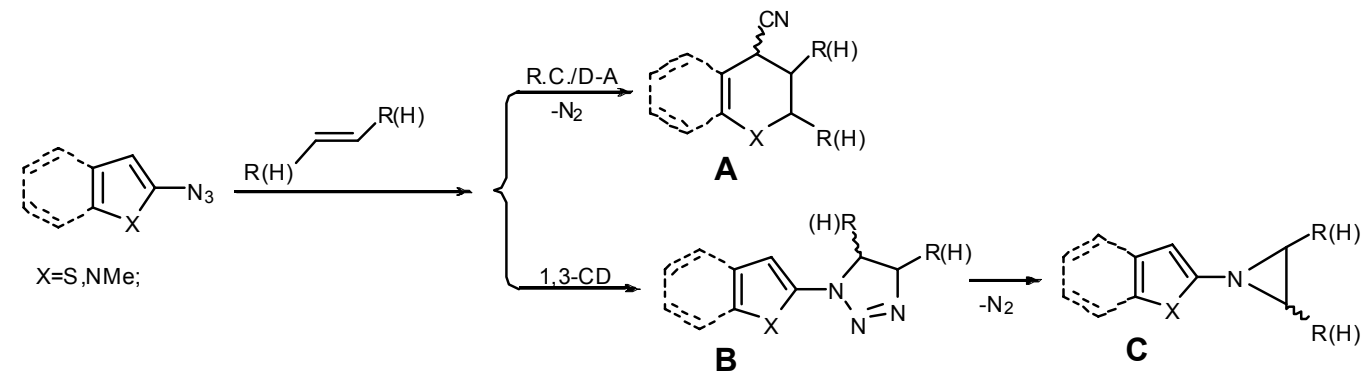

\section{Scheme 26}


This implies that the final overall product distributions strongly depend on the type of the heteroaryl, the nature of the alkene substituents and the reaction temperature, wherein the ring cleavage competes with the 1,3-DC. A similar trend in product distribution is observed for 2BTA and 2-azido- $N$-methylindole in reactions with a number of dipolarophile alkenes terminally, such as acrylonitrile, methyl acrylate, and trimethyl(vinyl)silane, or disubstituted, such as dimethyl- and diethyl-maleate, -fumarate, and $(E)$ and $(Z)$ methyl crotonate - that produce mixtures of regio- and stereo-specific 4-cyanothiochromans (4-51\%), 4cyanotetrahydroquinolines (8-46\%), and trans- and cis-1-(2-benzothienyl)- and 1-(Nmethylindol-2-yl)-aziridines (34-90\% and 14-72\%, respectively) as the major products. ${ }^{14,16}$

Later we succeeded in gaining clear-cut evidence for the intermediacy of an elusive 1,2,3triazoline in the formation of the aziridines when the 1,3-DCs of 2-BTA and 3-BTA with diethylfumarate were carried out at -20 and $5^{\circ} \mathrm{C}$. The $\alpha$-diazoamines $\mathbf{A}(30-40 \%)-$ diagnostic for the formation of aziridines at the expense of triazolines - are mainly formed under these conditions. (see Scheme 27) Similar results are obtained from 1,3-DC of 2-BTA and 3-BTA, and the dipolarophile methyl acrylate for reactions carried out at room and low temperatures. In these instances, the resulting $\alpha$-diazoamine (A, Scheme 27) structures [namely: ethyl-3(benzo $[b]$ thiophen-2 and 3-ylamino)-2-diazopropanoates] account for the regioselectivity of the $1,3-\mathrm{DCs}{ }^{48}$

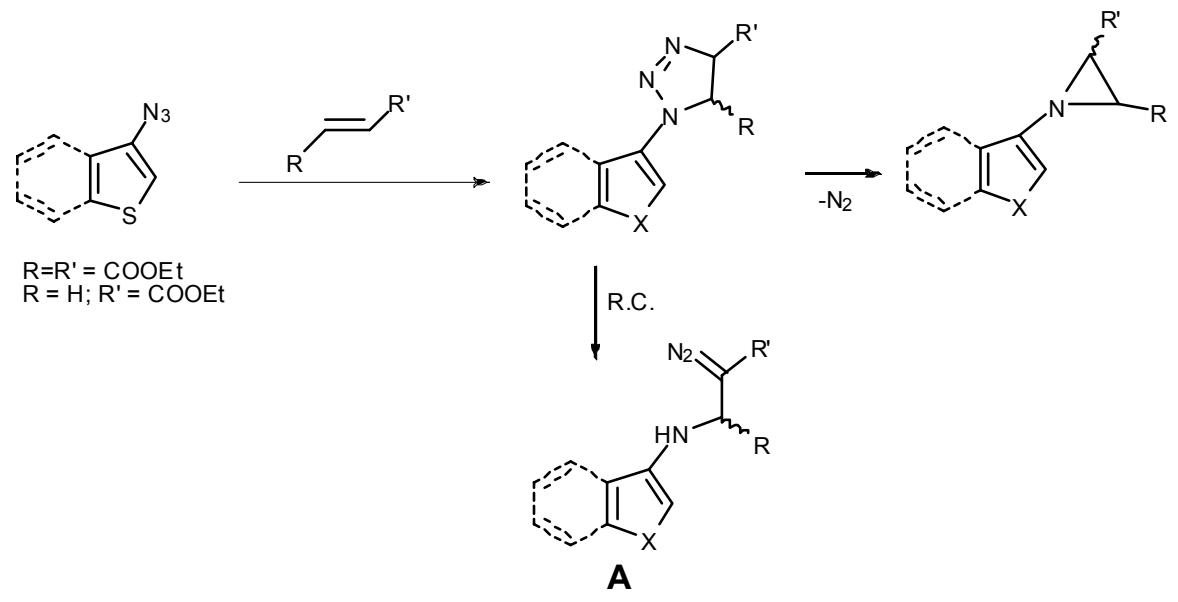

\section{Scheme 27}

In contrast, $\mathrm{C}^{\prime}$ '-pyrrolidinyl 1,2,3-triazolines are quickly obtained in quantitative yields (92-99\%) by 1,3-DCs of 2-BTA, 3-BTA, and 2-azido- $N$-methylindole with equivalent amounts of 'activated' alkenes, such as 1-pyrrolidinyl-cyclopentene and-cyclohexene in benzene. ${ }^{14,48}$ (see Scheme 28) 


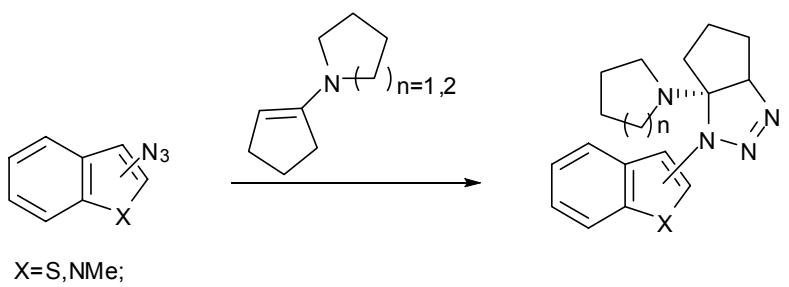

\section{Scheme 28}

These remarkable outcomes represent the first examples of stable, regiospecifically obtained 1,2,3-triazolines that bear a heteroaryl substituent at the $\mathrm{N}-1$ atom. A theoretical study, based on frontier molecular orbital/Pearson's hard-soft acid-base (FMO/HSAB) principles, ${ }^{49,50}$ confirms the high regiospecificity of the resulting 5-pyrrolidinyl-1-heteroaryl 1,2,3-triazolines. ${ }^{14}$

It would be useful to have a general 1,3-DC method based on a single dipolarophile alkene that acts as a solvent/co-reagent at room temperature with a wide range of aryl and heteroaryl azides. We carried out 1,3-DC studies using excess trimethylvinylsilane (TMVS) as a dipolarophile with a series of aryl azides. The standard reactions are classified by the nature of the azido group, and indicate that the phenyl azides carrying $p$-conjugated electron-withdrawing groups react about six times faster than those carrying electron-donating groups. ${ }^{51 \mathrm{~b}}$ Corresponding behaviour is found with electron-poor $p$-nitro and $p$-cyano phenyl azides, whose cycloadditions mainly afford C-4' trimethylsilylated 1,2,3-triazolines (95 and 87\%, respectively), which are quickly converted at $70^{\circ} \mathrm{C}$ in the corresponding aziridines. Otherwise, the minor reactivity of the azido group in the instances of the phenyl azide and electron-rich phenyl azides (40-65 days) results in longer reaction times. This change highly favours the formation of mixtures of 2-trimethylsilyl aziridines $\mathbf{A}$ and 3,5-bis(trimethylsilyl)pyrazolines $\mathbf{B}$, whose ratios were found to depend on the nature of the substituent. ${ }^{51 a}$ (Scheme 29)

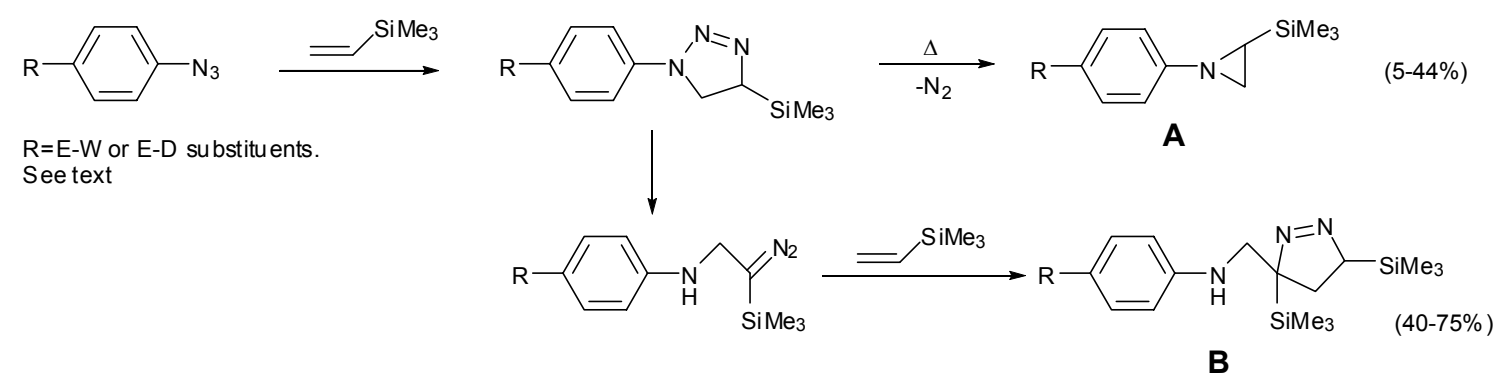

\section{Scheme 29}

The formation of the pyrazolines B proceeds via the ring cleavage of the initially formed triazoline ring, which generates 1,3-dipolar $\alpha$-diazoamines that are then trapped by the excess TMVS. Under the same conditions, 2-azido and 3-azido-thiophene, ${ }^{33}$-azido and 3-azidoselenophene, ${ }^{12}$ and 2-BTA and 3 -BTA ${ }^{51 a}$ with TMVS produce the corresponding 2- 
trimethylsilyl aziridines, respectively, in 75, 82, 77, 82, 93 and 95\% yields, but at different time rates $(4,12,3,11,5$, and 15 days, respectively).

Other fruitful and regioselective azido 1,3-DCs performed at room temperature are:

i) the reaction of azidoheteroaryls with equivalent amounts of methyl 3- $(N-$ pyrrolidinyl)acrylate (MePA) ${ }^{52}$ in an inert solvent to form methyl 1-(2- or 3-heteroaryl)triazole4-carboxylates with over $91 \%$ yields. The aromatisation to triazole rings proceeds by the elimination of the pyrrolidine from the initial triazolines.

ii) the reaction of aryl azides and heteroaryl azides in the presence of the carbon-carbon triple bonds (trimethylsilyl)acetylene (TMSAc) as reagents/solvents to the corresponding C-4 trimethylsilyl 1,2,3-triazoles. ${ }^{51 \mathrm{a}}$

The ease and useful reaction with TMSAc to 1,2,3-triazoles deserves some consideration. All of the 2-azido- and 3-azido-heteroaryl previously prepared are used for 1,3-DCs in the presence of TMSAc, as a facile source of novel biheteroaryls. ${ }^{12,14,22,32,33,53}$

It must be emphasised once again that the TMSAc 1,3-DCs carried out at low to room temperatures can be considered a useful test of azido-reactivity, especially for the instable 2azidoheteroaryls. The additions are generally regiospecific and give $\mathrm{C}-4$ ' trimethylsilyl 1,2,3triazoles as the main products in the case of unstable 2-azidoheteroaryl derivatives, such as 2azido- $N$-methylindole $(60 \%),{ }^{14}$ 2-azidothiophene $(75 \%){ }^{33} \quad$ 2-azido-1-methyl- $1 H$-imidazole $(76 \%),{ }^{22}$ and 3 -azido- $N$-methylindole $(81 \%),{ }^{14}$ whereas more stable starting azides give C-4' substituted triazoles in higher yields, for example 2-azidoselenophene $(83 \%){ }^{12} 3$ azidoselenophene (87\%), ${ }^{12}$-azido-1-methyl- $1 H$-imidazole $(88 \%),{ }^{22} 3$-azidothiophene $(90 \%){ }^{33}$ 2-azido-1,3-thiazole $(90 \%){ }^{22}$ 2-azidobenzo-1,3-thiazole $(90 \%){ }^{22}$ 2-BTA $(93 \%)$ and 3-BTA $(96 \%),{ }^{51}$ and 5-azido-1-methyl-1H-pyrazole $(98 \%) .^{22}$ Owing to the practical applications of 1,2,3-triazoles in agriculture and medicine, ${ }^{54}$ several of these compounds have been submitted to the National Cancer Institute (NCI's anti-Cancer) and the National Institute of Allergy and infectious Diseases (NIAID's anti-Tuberculosis) drug discovery program with promising results on primary and in vitro assays. ${ }^{55}$

The 1,3-DC times of electron-poor phenyl azides are twofold faster with the $\mathrm{C}$-C double bonded TMVS compared to that of the C-C triple bonded TMSAc. On the other hand, reaction times with electron-rich phenyl azides are comparable for both of the dipolarophiles. These findings indicate that the mechanism is clearly dominated by $\mathrm{HOMO}_{\text {dipole }} / \mathrm{LUMO}_{\text {dipolarophile }}$ interaction. The electron poor azides are more discriminating towards the lower nucleophilic nature of the alkyne with respect to the corresponding alkene. Incidentally, the rate of the cycloadditions of aryl azides with TMSAc to form the corresponding 1,2,3-triazoles — when measured by FTIR in a diamond anvil cell - increases logarithmically with pressure (up to 1 $\mathrm{GPa}) .{ }^{56}$

Recently, there has been great interest in the 1,3-DCs reaction of organic azides and alkynes to form regiospecific C-4' substituted 1,2,3-triazoles. ${ }^{57}$ Two independent papers on this topic from Meldal and Sharpless — who have reported the use of a copper (I) catalyst in 1,4regioselective 1,3-DCs - are significant examples. ${ }^{58}$ 


\section{Five-membered Azido(heteroaryl)methanones}

\subsection{General synthesis and Curtius rearrangement}

The thermally promoted 1,2-migration of a carbon atom of the acyl azide and the contemporary $\mathrm{C}-\mathrm{C}$ bond shift into the $\mathrm{C}-\mathrm{N}$ bond with elimination of molecular nitrogen to isocyanates, is called Curtius rearrangement (C.R.) (Scheme 30). In general the process is concerted, but, depending on the substrate, it sometimes takes place via a 'free' nitrene intermediate involving a two or three step mechanism. ${ }^{3 a, b, 59}$ When carried out under dry conditions, the thermal reaction of the azido(heteroaryl)methanones (heteroaroyl azides) can be considered the most convenient route to prepare large amounts of heteroaryl isocyanates and diisocyanates, with no use of the phosgene. ${ }^{60}$ (see Scheme 30)

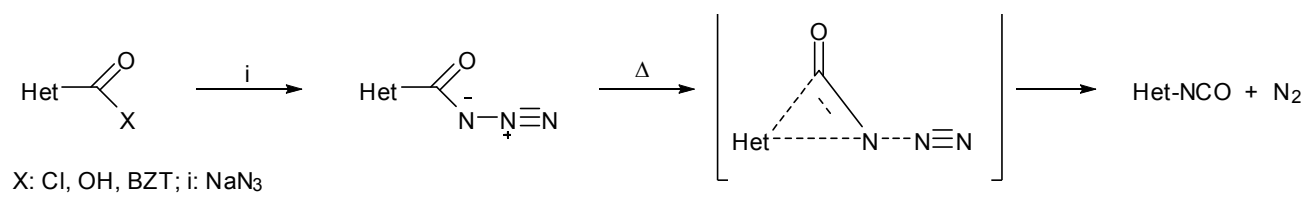

\section{Scheme 30}

Since isocyanates are sensitive to a great variety of reagents, such as alcohols, amines, enamines, and aromatic and organometallic compounds, the entire process could be regarded as the conversion of inexpensive heteroaryl carboxylic acids into the corresponding amines or their derivatives like urethanes, ureas, carboxamides, $\beta$-lactams, and carbamates. ${ }^{61}$

Differential scanning calorimetry (DSC) analysis with azido(heteroaryl)methanones has been performed to provide information about their thermochemical conversions and classification as high-energetic materials. In this study, 2-furyl 1, 2-thienyl 2, 5-methyl-2-thienyl 3, 5-(trimethylsilyl)-2-thienyl 4, and 2-selenophenyl-azidomethanone 5 were converted into the appropriate isocyanates, or their secondary products, under a set of standard conditions. Handling compounds 1-5 appears safe on the basis of the thermodynamic evaluation of their energetic releases. $^{2 \mathrm{~b}, 62}$

The starting azido(heteroaryl)methanones 1-5 are normally prepared from heteroaryl carboxylic acids and ethyl chloroformate via mixed carboxylic-carbonic anhydrides and sodium azide $^{63}$ or the treatment of an acyl chloride with trimethylsilyl azide, ${ }^{64}$ instead of the more hazardous displacement reaction of acyl chloride with the azido ion. ${ }^{65}$ Recently, a general and safe synthesis of acyl azides, including azido(2-thienyl and 2-furyl)methanones (80 and 75\% yield, respectively), from the corresponding $N$-acyl benzotriazoles and sodium azide has been reported. $^{66}$

DSC-thermolysis of compounds $\mathbf{1}-\mathbf{5}$ at $30-220^{\circ} \mathrm{C}$ in dry hexadecane for 19 min gives the corresponding isocyanates, with the sole exception of the unstable azide 4 , which affords a isocyanate $\mathbf{2}$ by concomitant desilylation (Scheme 31). Otherwise, the azides $\mathbf{1}-\mathbf{3}$ and $\mathbf{5}$ undergo 
similar thermal decomposition in 1-dodecanol, giving the appropriate heteroaryl carbamates in high yields ( $>92 \%$ ), while the azide 5 results in a lower yield $(56 \%)$, presumably due to the lesser stability of the final carbamate. ${ }^{2 b}$

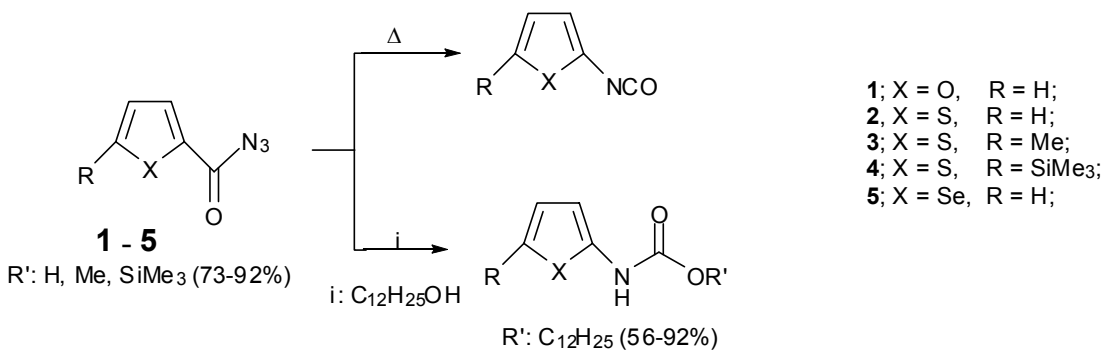

\section{Scheme 31}

Room temperature reactions between three heteroaroyl chlorides - namely, 2-thienoyl-, 3thienoyl- and 2-benzo[b]thienoyl-chloride - with trimethylsilyl azide (TMSA) essentially yielded the corresponding azido(2-thienyl, 3-thienyl, and benzo[b]thienyl)methanones. (see Scheme 32, path I, Het = 2-thienyl, 3-thienyl, and 2-benzo[b]thienyl) These acyl azides are converted in fairly good yields $(52,70$, and $79 \%$, respectively) into the appropriate isocyanates when heated in a sealed tube at $90^{\circ} \mathrm{C}$ for $1 \mathrm{~h}$ (Scheme 32, path I). On the other hand, in refluxing $\mathrm{CCl}_{4}$ with excess TMSA, the same reaction preferentially yields the 1,4-disubstituted heteroaryl(trimethylsilyl)tetrazol-5-ones $\mathbf{A}$; through regioselective azido 1,3-DC addition of the 1,3-dipolar azido group of the TMSA to the N-C bond of the former heteroaryl isocyanates. ${ }^{64}$ (see Scheme 32, path II)

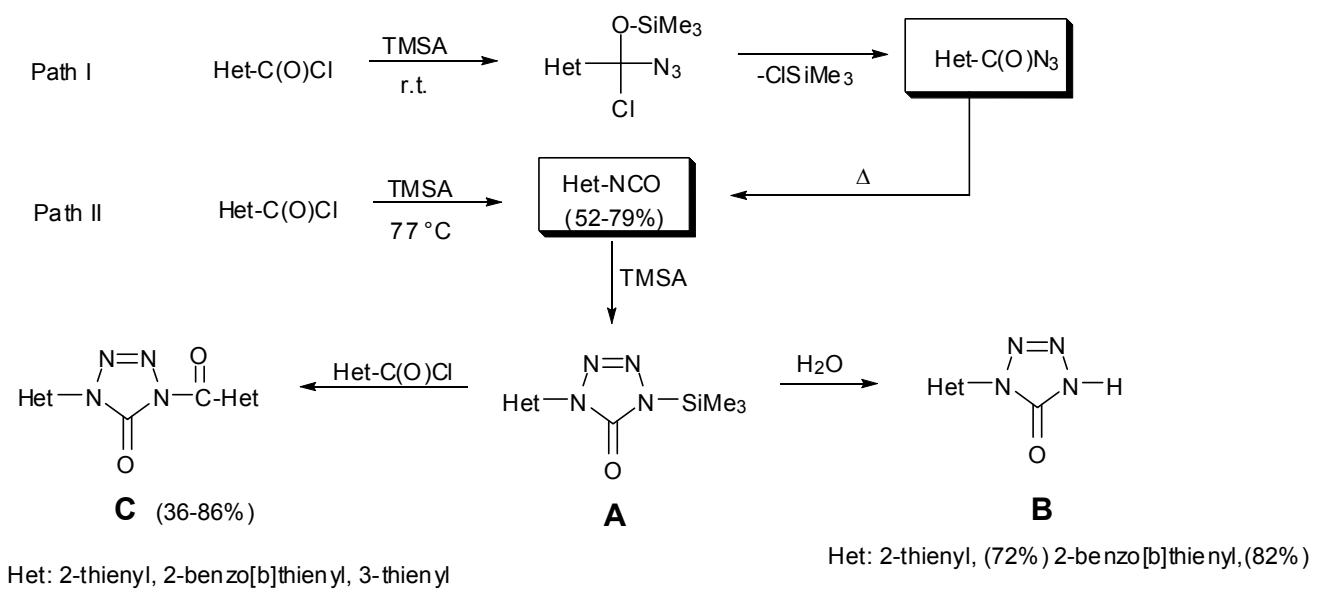

\section{Scheme 32}

Treatment of the resulting 1-heteroaryl-4-(trimethylsilyl) tetrazol-5-ones A with water gives the corresponding desilylated 1-heteroaryl tetrazol-5-ones $\mathbf{B}$; via electrophilic substitution at the 
nitrogen N-4. For other conversions, the tetrazol-5-ones A react with an equimolar amount of the heteroaroyl chlorides, leading to the 1-heteroaroyl-4-heteroaryltetrazol-5-ones C. (see Scheme 32)

If the thermal decomposition of azidomethanones 1-5 plus the azido[5-(trimethylsilyl)-2selenophene]methanone 6 is carried out at $90^{\circ} \mathrm{C}$ in the presence of neat $N$-methylpyrrole, then the main products are $N$-linked 2-heteroaryl-1-methylpyrrole-2-carboxamides $\mathbf{1 b}-\mathbf{6} \mathbf{b}$ at a yield of $68-83 \%$ via direct attack of isocyanates $1 \mathbf{a}-\mathbf{6 a}$ on the pyrrole ring. ${ }^{67}$ (see Scheme 33 ) The resulting compounds $\mathbf{1 b}-\mathbf{6 b}$ are of special interest as a potential nitrogen mustard of the diastomycin type, ${ }^{68}$ while the heteroaryl amide linkage can play a synergistic role in bioactivity. $^{69}$

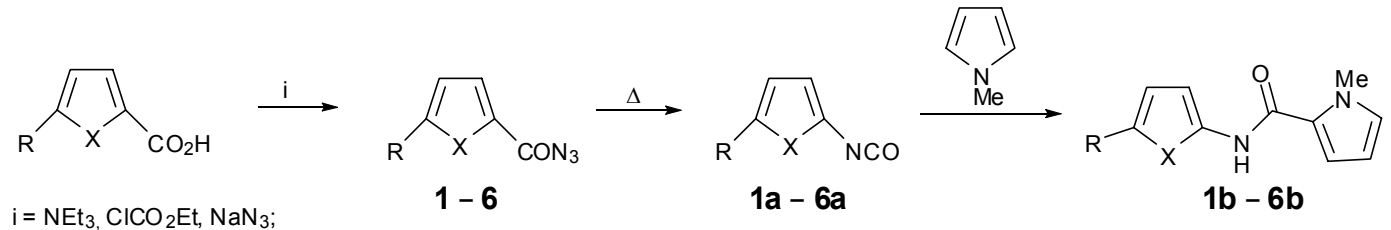

\section{Scheme 33}

\subsection{1,3-Dipolar cycloadditions involving azido(heteroaryl)methanones}

Another intriguing topic in 1,3-DCs involving organic azides is represented by the reactions of the azido(heteroaryl)methanones with dipolarophiles; however, few examples of intramolecular reactions have been reported probably due to the lesser reactivity of the deactivated acylazido component. $^{70}$ To our knowledge, no report has described the intermolecular 1,3-DCs of the azido component of azido(heteroaryl)methanones with dipolarophiles as a possible route to acyl triazoles. Related examples would be the known 1,3-DCs involving arenesulphonyl azide and ethyl azidoformate with strained dipolarophiles. ${ }^{59 a}$

Effectively, we provide evidence for the applicability of 1,3-DC to the synthesis of cyclic azoles using some azido(heteroaryl)methanones with 'activated' olefins, such as methyl (E)-3( $N$-pyrrolidinyl)acrylate (MePA) or 'strained' ones, such as 5-methylenebicyclo[2,2,1]hept-2-ene (MBH), but not with electron-poor dipolarophiles, such as methyl crotonate and methyl propiolate, or 'deactivated' methyl but-2-ynoate. ${ }^{71}$

In the case of MePA, the room temperature reaction of equimolar amounts of the acyl azides, namely azido(2-furyl, 2-thienyl, 5-methyl-2-thienyl, 2-selenophenyl and thieno[3,2b] thiophen-2-yl) methanone, in $\mathrm{CDCl}_{3}$ gives a mixture of triazole-4-carboxylate $\mathbf{A}$ and $N$ pyrrolidinyl(heteroaryl)methanone B after 20-25 days. (see Scheme 34) The reaction occurs by loss of pyrrolidine base from the former 1,3-cycloadduct, finally resulting in the formation of triazole-4-carboxylate and pyrrolidinyl(heteroaryl)methanone. The liberated pyrrolidine base attacks the carbonyl carbon via the nucleophilic addition of the former cycloadduct, producing a disubstituted heteroarylmethanol intermediate $\mathbf{I}$, which in turn, releases the two final products. 


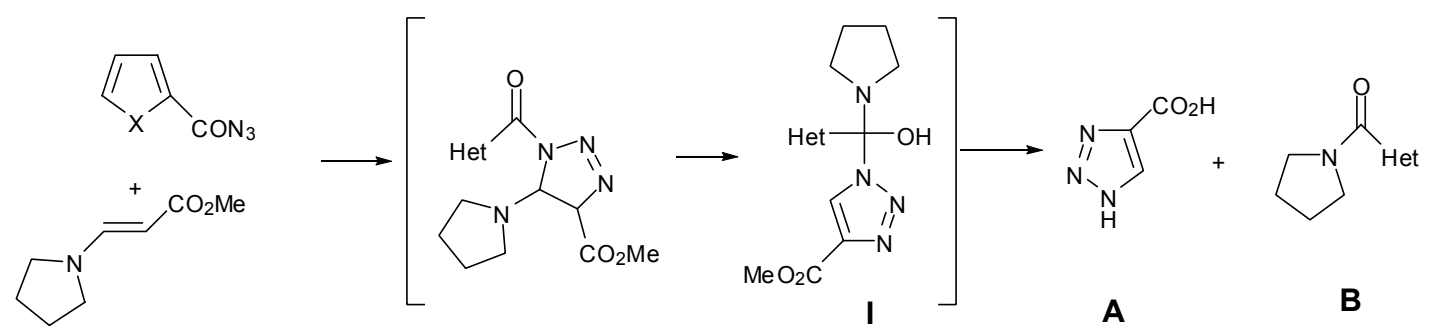

\section{Scheme 34}

An interesting case arises when similar 1,3-DCs of the same azido(heteroaryl)methanones are performed in the presence of $\mathbf{M B H}$, which in principle contains two reactive double bonds. In such a case, the strained endo double bond is greatly favoured, and the cycloadditions occur at room temperature to give the aziridine compounds $\mathbf{A}$, via unstable isomeric 1,2,3-triazoline adducts I that easily lose molecular nitrogen instantaneously with ring contraction. (see Scheme 35)

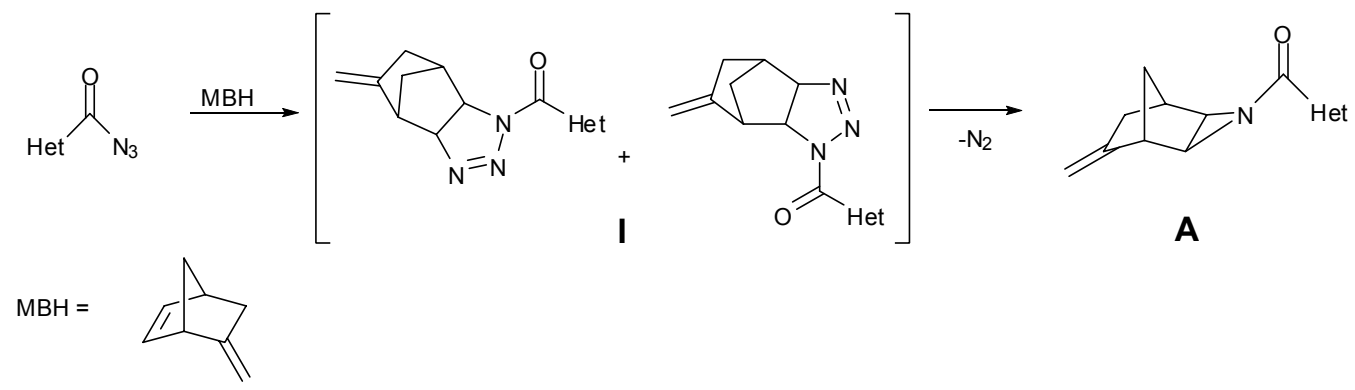

\section{Scheme 35}

Experiments carried out with ${ }^{1} \mathrm{H}$ NMR spectroscopy provide evidence for the fast conversion of the triazoline adduct $\mathbf{I}$ to ring-fused heteroarylcarbonyl aziridines $\mathbf{A}$. Similar triazoline ring contraction to aziridines have previously been observed in the case of 1,3-DC of benzoyl azide and ethyl azidoformate with norbornene. ${ }^{59 a}$

\section{Conclusions and Outlook}

The successful use of the 'azido transfer' protocol greatly improves the impact of novel molecules in the field of nitrogen-linked heteroaryl systems. The number of available heteroaryl azides has increased considerably, and is likely to continue to grow, in view of the overwhelming number of potential heteroaryl candidates. In many instances, the protocol facilitates the easy transformation of heteroaryl-lithium derivatives into various nitrogen-linked heteroaryl 
compounds, such as amines, triazenes, triazolines, aziridines, triazoles, or six-membered novel heterocycles, whose emerging chemistry is still yet to be fully discovered. ${ }^{72,73}$

In light of the present evidence, it can be concluded that the peculiar unimolecular thermal fragmentation of the 2-azidoheteroaryls is likely to proceed via a low-energy process involving concerted ring cleavage with the loss of nitrogen, rather than via a 'free nitrene' intermediate as for the phenyl azides. The resulting 4-cyano 1,3-heterodienes can be trapped via [4+2]cycloaddition with dienophiles to form six-member heterocycles. Moreover, both 2-azido- and 3azido-heteroaryls normally react with a great variety of dipolarophile alkynes and alkenes in a fashion slightly different from that of the phenyl azides, in several cases, mainly giving 1,2,3triazole or aziridine, respectively, via 1,2,3-triazoline-biheteroaryls. The low stability observed for the N-1 substituted 1,2.3-triazoline-biheteroaryls probably deserves chemical-biological interface research. In addition, more stable 1,2,3-triazolines are favoured with electronwithdrawing substituents located on the $\mathrm{N}-1$ atom or electron-donor groups located on the triazoline ring. Otherwise, the room temperature 1,3-DCs of the heteroaryl azides with (trimethylsilyl)acetylene or methyl 3-( $N$-pyrrolidinyl)acrylate afford stable 1-(2-heteroaryl or 3heteroaryl)-1,2,3-triazoles C-4'-trimethylsilyl or C-4'-methylcarboxylate, which can be employed as novel antifungal and antihelmintic compounds.

Finally, the chemistry of these compounds is also derived from azido(heteroaryl)methanones since they are facile precursors of heteroaryl isocyanates (or diisocyanates), which are obtained with no use of the phosgene, as well as azido(heteroaryl)methanones, which can react directly, under mild conditions, with alcohols, amines, and many other reagents. These reactions will have a significant impact on the production of modern pharmaceutical agents, biologically active molecules, or a range of industrial polymers for high-technology applications., ${ }^{4,60}$

\section{Acknowledgements}

The author is grateful to the Royal Society of Chemistry for a Journal Grant for the Authors (Application $\mathrm{N}^{\mathrm{o}} 0710$ 596), who were able to attend at the $9^{\text {th }}$ FloHet 2008 at Gainesville. Gratefully acknowledge to the 'Ministero della Università e Ricerca' for the main financial support (60\% and PRIN 2005 and 2007) and the Ateneo di Bologna from the 'Progetto di Finanziamento Triennale'. The Author takes this opportunity to express his deep gratitude to all the co-workers cited in the related references.

\section{References and Notes}

1. Bretherick's Handbook of Reactive Chemical Hazards, Urben, P. G. Ed.; Elsevier: Amsterdam, $7^{\text {th }}$ Ed., 2007; Vols. 1 and 2. Agrawal, J. P.; Hodgson, R. D. In Organic 
Chemistry of Explosives, Wiley: Chicester, 2007. Huynh M.-H. V.; Hiskey M. A.; Chavez D. E.; Naud D. L.; Gilardi R. D. J. Am. Chem. Soc. 2005, 127, 12537.

2. (a) Cardillo, P.; Gigante, L.; Lunghi, A.; Fraleoni-Morgera, A., Zanirato, P. New J. Chem. 2008, 32, 47. (b) Salatelli, E.; Zanirato, P. ARKIVOC 2002, (xi), 6. (c) Stadlbauer, W.; Hojas, G. J. Biochem. Biophys. Methods 2002, 53, 89.

3. (a) Smith, P. A. S. Aryl and Heteroaryl Azides and Nitrenes In Azides and Nitrenes, Reactivity and Utility; Scriven, E. F. V., Ed., Academic: Orlando, 1984; Chap. 3. (b) Scriven, E. F. V.; Turnbull, K. Chem. Rev. 1988, 88, 298. (c) Brase, S.; Gil, C.; Knepper, K.; Zimmermann, V. Angew. Chem. Int. Ed. 2005, 44, 5188. (d) Fraleoni-Morgera, A.; Zanirato, P. ARKIVOC 2006, (xii), 111. (e) Schuster, G. B.; Platz, M. S. Adv. Photochem. 1992, 17, 69. (f) Hrovat, D. A.; Waali, E. E.; Thatcher Borden, W. J. Am. Chem. Soc. 1992, 114, 8698. (g) Albini, A.; Bettinetti, G.; Minoli, G. J. Chem. Soc. Perkin 1 1999, 2803. (h) Kvaskoff, D.; Bednarek, P; George, L.; Pankajakshan, S.; Wentrup, C. J. Org. Chem. 2005, 70, 7947.

4. Evans, R.A. Aus. J. Chem. 2007, 60, 384-395. Aziridine and Epoxides In Organic Synthesis; Yudin A. K. Ed.; Wiley-VCH: Weinheim, 2006. Falvey, D. E. In Reaction Intermediate Chemistry; Moss, R. A.; Platz, M. S.; Jones, M. Jr Eds.; Wiley-VCH: Weinheim, 2004; 593650. Caselli, A.; Gallo, E.; Fantauzzi, S.; Morlacchi, S.K; Ragaini, F.; Cenini, S. Eur. J. Inorg. Chem. 2008, 3009. Pinho e Melo, T. M. V. D.; d'A. Rocha Gonsalves, A. M. Curr. Org. Synthesis 2004, 1, 275.

5. Smith, P. A. S.; Rowe, C. D.; Bruner, L. B. J. Org. Chem. 1969, 34, 3430.

6. (a) Spagnolo, P.; Zanirato, P. J. Org. Chem. 1978, 43, 3539-3541. (b) Spagnolo, P.; Zanirato, P. J. Chem. Soc., Perkin 1 1988, 3375. (c) Toselli, M.; Spagnolo, P.; Zanirato, P. Gazz. Chim. It. 1989, 119, 411.

7. Chinchilla, R.; Najera, C.; Yus, M.; ARKIVOC 2007, (x), 152. Clayden, J. In Organolithium Selectivity for Synthesis; Pergamon: Amsterdam, 2002. Wakefield B. J. Organolithium methods In Best Synthetic Methods; Katritzky, A. R.; Meth-Cohn, O.; Rees, C. W. Eds.; Academic: Orlando, 1988.

8. Khramov, D. M.; Bielawski, C. W. Chem. Commun. 2005, 4958. Saunders K. H., The Aromatic Diazo Compounds; Longmans: New York, $2^{\text {nd }}$ edn, 1949.

9. Rouzer, C. A.; Sabourin, M.; Skinner, T. L.; Thompson, E. J.; Wood, T. O.; Chmurny, G. N.; Klose, J. R.; Roman, J. M.; Smith, R. H. Jr; Michejda, C. J. Chem. Res. Toxicol. 1996, 9, 172.

10. See for sample: Lippert, T.; David, C.; Hauer, M.; Wokaun, A.; Robert, J.; Nuyken, O.; Phipps, C. J. Photochem. Photobiology A: Chem. 2001, 145, 87 and refs therein.

11. For a monograph on this topic see: Biffin, M. E. C.; Miller, J.; Paul, D. B. Introduction of the Azido Group, In The Chemistry of the Azido Group; Patai S. Ed., Wiley: New York; Ch 2, 1971. For a recent review on this topic see also Ref. 3c.

12. Gronowitz, S.; Zanirato, P. J. Chem. Soc. Perkin 1 1994, 1815.

13. Nanni, D.; Zanirato, P. J. Chem. Soc. Perkin 1 1997, 1003.

14. Foresti, E.; Di Gioia, M. T.; Nanni, D.; Zanirato, P. Gazz. Chim. It. 1995, 125, 151. 
15. Foresti, E.; Spagnolo, P.; Zanirato, P. J. Chem. Soc., Perkin 1 1989, 1354.

16. Spagnolo, P.; Zanirato, P. J. Chem. Soc., Chem. Commun. 1985, 1441.

17. (a) Davies, D.; Spagnolo, P.; Zanirato, P. J. Chem. Soc., Perkin 1 1995, 613. (b) Spagnolo, P.; Zanirato, P. J. Chem. Soc., Perkin 1 1996, 963.

18. For a review on the topic see: Dehan, W.; Becher, J. Acta Chem. Scand. 1993, 47, 244.

19. Faure, R.; Galy, J. P.; Vincent, E. J.; Elguero, J. Can. J. Chem. 1978, 56, 46.

20. Cubero, E.; Orozco, O.; Luque, F. J. J. Am. Chem. Soc. 1998, 120, 4723.

21. Granados, R.; Rull, M.; Vilarrasa, J. J. Heterocycl. Chem. 1976, 13, 281. Faure, R.; Galy, J. P.; Vincent, E. J.; Elguero, J. J. Heterocycl Chem. 1977, 14, 1299.

22. Cerini, S.; Zanirato, P.Org. Biomol. Chem. 2005, 3, 1508.

23. Jutzi, P.; Sacriß, W. Chem. Ber. 1973, 106, 2815.

24. Dondoni, A.; Fantin, G.; Fogagnolo, M.; Medici, A.; Pedrini, P. J. Org. Chem. 1988, 53, 1748 .

25. Gilman, H.; Beel, J. A. J. Am. Chem. Soc. 1949, 71, 2328.

26. Katritzky, A. R.; Jayaram, C. S.; Vassilatos, N. Tetrahedron 1983, 39, 2023.

27. For a series of reviews on lithiated azoles see: Iddon, B.; Ngochindo, R. I. Heterocycles 1994, 38, 2487. Iddon, B. Heterocycles 1995, 41, 533. Grimmet, M. R.; Iddon, B. Heterocycles 1994, 37, 2087.

28. Blankenship, C.; Wells, G. J.; Paquette, L. A. Tetrahedron 1988, 44, 4023.

29. Funicello, M.; Spagnolo, P.; Zanirato, P. Acta Chem. Scand. 1993, 47, 231.

30. (a) Valenti, F.; Zanirato, P. J. Chem. Soc., Perkin 2 1999, 623. (b) Zanirato, P. Gazz. Chim. It. $1997,127,339$.

31. (a) Amantini, D.; Fringuelli, F.; Pizzo, F.; Vaccaro, L. Org. Prep. Proc. Int. 2002, 34, 111.

(b) Gronowitz, S.; Westerlund, C.; Hornfeldt, A.-B. Acta Chem. Scand. 1975, B29, 224.

32. Gronowitz, S.; Spagnolo, P.; Zanirato, P. J. Org. Chem. 1982, 47, 3177.

33. Spinelli, D.; Zanirato, P. J. Chem. Soc. Perkin 1 1993, 1129.

34. Spagnolo, P.; Zanirato, P. J. Chem. Soc. Perkin 1 1988, 3375.

35. Abramovitch, R. A.; Kyba, P.; Scriven, E. F. V. J. Org. Chem. 1971, 36, 3796 and refs therein.

36. (a) Smith, P. A. S.; Brown, B. B. J. Am. Chem. Soc. 1951, 73, 2438. (b) Smith, P.A.S.; Hall, J. H. J. Am. Chem. Soc. 1962, 84, 480.

37. (a) Dyall, L. K.; Kemp, J. E. J. Chem. Soc. B 1968, 976. (b) Lindley, J. M.; McRobbie, I.; Meth-Cohn, M. O.; Suschitzky, H. J. Chem. Soc., Perkin 1 1977, 2194. (c) Smolinsky, G. J. Am. Chem. Soc 1961, 83, 2489. (d) Ref. 3a.

38. Spagnolo, P.; Zanardi, G.; Zanirato, P. J. Chem. Soc., Perkin 1 1983, 2551.

39. For a review on the topics see: Abramovitch, R. A.; Kyba, E. P. Decomposition of Organic Azides, In The Chemistry of the Azido Group; Patai, S., Ed.; Interscience: New York, 1971; Ch 5, pp 221-329.

40. Takeuchi, H.; Takano, K. J. Chem. Soc., Chem. Commun. 1983, 447 and refs therein. 
41. (a) Zanirato, P. J. Chem. Soc. Chem. Commun. 1983, 1065. (b) Spagnolo, P.; Zanirato, P. J. Chem. Soc., Perkin 1 1988, 2615.

42. Falvey, D. E. J. Phys. Org. Chem. 1999, 12, 589.

43. Spagnolo, P.; Zanirato, P. Tetrahedron Lett. 1987, 28, 961; Ref. 3 b.

44. Zanirato P. J. Organomet. Chem. 1985, 293, 285.

45. Leardini, R.; Zanirato, P. J. Chem. Soc., Chem. Commun. 1983, 396.

46. Gronowitz, S.; Ander, I.; Zanirato, P. Chem. Scripta 1983, 22, 55.

47. March, J.; Smith, M. B. In Advanced Organic Chemistry; Wiley: New York; 2007, $5^{\text {th }}$ Ed., pp 1059-1062. Padwa, A. In 1,3-Dipolar Cycloaddition Chemistry; Wiley: New York; 1984, Vol. 2.

48. Funicello, M.; Spagnolo, P.; Zanirato, P. J. Chem. Soc., Perkin 1 1990, 2971.

49. Chandra, A. K.; Uchimaru, T.; Nguyen, M. T. J. Chem. Soc., Perkin 1 1999, 2117.

50. Fleming, I. In Frontier Orbital and Organic Chemical Reactions; Wiley: London, 1976. Huisgen, R. J. Org. Chem. 1976, 41, 403. Halevi, E. A. In Orbital Symmetry and Mechanism. The OCAMS view; Springer-Verlag: Heidelberg, 1992; pp 169-170.

51. (a) Zanirato, P. J. Chem. Soc., Perkin 1 1991, 2789. (b) Funicello, M.; Zanirato, P. Gazz. Chim. It. 1995, 125, 151.

52. Al-Sader, B. H.; Kadri, M. Tetrahedron Lett. 1985, 26, 4661.

53. Wamhoff, H. In Comprehensive Heterocycl. Chem.; A. R. Katritzky, A. R.: Rees, C. W.; Potts, K. T., Eds. Pergamon: Oxford, 1984, 5, Part 4.A.

54. Businelli, S., Syntheses and Structures of Bioactive Biheteroaryls, Undergraduate Thesis, University of Bologna, 1997.

55. Businelli, S.; Di Martino, E.; Zanirato, P. ARKIVOC 2001, (ii), 131.

56. Melai, V.; Brillante, A.; Zanirato, P. J. Chem. Soc., Perkin 2 1998, 2447.

57. Tron, G. C.; Pirali, T.; Billington, R. A.; Canonico, P. L.; Sorba, G.; Genazzani, A. A Med. Res. Rev. 2008, 28, 278. Moses, J.; Moorhouse, A. D. Chem. Soc. Rev. 2007, 36, 1249. Evans, R. A.; Austral. J. Chem. 2007, 60, 384. Katritzky, A. R.; Zhang, Y.; Sin, S. Heterocycles 2003, 60, 1225.

58. Rostovtsev, L. G.; Green, L. G.; Fokin, V. V.; Sharpless, K. B. Angew. Chem. Int. Ed. 2002, 41, 2596. Tornoe, C. W.; Christensen, C.; Meldal, M. J. Org. Chem. 2002, 67, 3057.

59. (a) Lwowski, W. Acyl Azides and Nitrenes In Azides and Nitrenes, Reactivity and Utility; Scriven, E. F. V. Ed.; Academic: Orlando, 1984, Ch. 3, pp 205-246. Acyl Azides In The Chemistry of the Azido Group, Patai, S., Ed., Wiley-Interscience: New York; Ch 9, pp 503554, 1971. (b) Moore, H.; Goldish, D. M. In Chemistry of Halides, Pseudo-Halides and Azides; Patai, S., Ed.,Wiley; Chichester; Ch. 1, 1983. (c) Edwards, O. E. In Nitrenes; Lwowski, W., Ed.; Interscience: New York, 1970.

60. LeBel, N. A.; Cherluck, R. M.; Curtius, E. A. Synthesis, 1973, 678-679. In Chemistry and Technology of Isocyanates; Ulrich, H., Ed; Wiley: Chichester, 1996. See also refs 1 and 3a.

61. Ziegler, T. Science of Synthesis 2005, 21, 43. 
62. Smith, P. A. S. In Organic Reactions, Wiley: New York, 1946, pp 337-349. See also Ref 1.

63. Weinstock, J. J. Org. Chem. 1961, 26, 3511.

64. Toselli, M.; Zanirato, P.; J. Chem. Soc., Perkin 2 1992, 1101.

65. Babu, V. V. S.; Ananda, K.; Vasanthakumar, G.-R. J. Chem. Soc., Perkin 2000, 4328.

66. Katritzky, A. R.; Widyan, K.; Kirichenko, K. J. Org. Chem. 2007, 72, 5802.

67. Danielli, F.; Zanirato, P. ARKIVOC 2000, (i), 58.

68. Gribble, G. W. In Comprehensive Heterocyclic Chemistry II; Katritzky, A. R.; Rees, C. W.; Scriven, E. F. V.; Storr, R. C. Eds.; Pergamon: Oxford, 1996.

69. Damayanthi, Y.; Reddy, B. S. P.; Lown, J. W. J. Org. Chem. 1999, 64, 290. Christiansen, L. E. E. In Selenophenes, ref. 68. Russel R. K.; Press, J. B. ref 68.

70. Broggini, G.; Garanti, ; Molteni, G.; Zecchi., G. J. Chem. Res., $(S)$ 1998, 688.

71. Zanirato, P., J. Chem. Soc., Perkin 1 2002, 1420.

72. Spinelli, D.; Zanirato, P. Chim. Ind., (Milan) 1996, 78, 953.

73. Katritzky, A. R.; Zhang, Y.; Sing, S. K.; Steel P. J. ARKIVOC 2003, (xv), 47. Sha, C.-K; Mohanakrishnan, A. K. Azides In Synthetic Applications of 1,3-Dipolar Cycloaddition Chemistry Toward Heterocycles and Natural Products In The Chemistry of Heterocyclic Compounds; Padwa, A.; Pearson, W. H. Eds; Wiley-Interscience: Published Online: 2003, Ch. 9, pp 623-679.

\section{Author's biographical data}

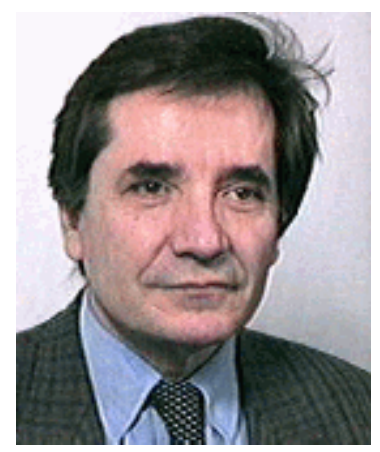

Paolo Zanirato received his doctor degree in 1972 from the Faculty of Industrial Chemistry at the Bologna University, where he was postdoctoral fellow until 1975. During the 1975-2009 lecturer in 'Synthetic Inorganic and Organic Chemistry', 'Experimental Organic Chemistry', 'Colour Chemistry', and 'Heterocyclic Chemistry' alternatively at the Faculty of Industrial Chemistry of Bologna and at the Faculty Pharmacy of the University of Chieti. In 1980 he became an Associate Professor of Organic Chemistry at the Faculty of Industrial Chemistry of the University of Bologna. Starting from 1972 the applicant has supervised numerous final year undergraduate research projects. He is a Fellow of the Royal Society of Chemistry (FRSC) and Member of the International Society of Heterocyclic Chemistry (MISHC) and has 83 papers to 
his credit, which to date include full research papers (67) mainly in the field of the heterocyclic compounds containing nitrogen functional groups, published on national and international journal, patent (1) and communication and oral/poster (25) presented at national and international conferences especially in the area of free-radical chemistry and heterocyclic chemistry. Starting from the academic year 2007-2008 the applicant has been responsible for the organization of a high-level course entitled 'Industrial safety and hazardous chemicals'.

\section{Field of specialisation}

The current research interests include synthetic, spectroscopic and mechanistic aspects of the chemistry of nitrogen-linked systems both carbo- and heterocyclic as well as molecular modelling on the structure-reactivity correlation. A strong emphasis has been placed on the synthesis of heteroaryl azido systems and on studies of their reactions under thermodynamic, kinetic and mechanistic points of view. 